\title{
Successive Interference Cancellation using Constellation Structure
}

\author{
Ananya Sen Gupta, Andrew Singer \\ University of Illinois at Urbana-Champaign \\ E-mail: $\{$ asengupt, acsinger $\} @$ ifp.uiuc.edu
}

\begin{abstract}
An approach to successive interference cancellation is presented that exploits the structure of the combined signal constellation in a multi-user system. The asymptotic conditional efficiency of a successive detector is defined, based on the conditional probability of error at high signal-to-noise ratio (SNR), as a quantitative measure for evaluating detector performance at each stage of successive detection. The joint successive interference canceller (JSIC) that jointly detects consecutive users in an ordered set is proposed as an improvement over the conventional successive interference canceller (SIC). The maximal asymptotic conditional efficiency successive interference canceller (MACE-SIC) and its JSIC equivalent (MACE-JSIC) are also derived as the multi-user detectors that achieve the highest asymptotic conditional multi-user efficiency at each stage of successive detection among all possible SIC and JSIC detectors, respectively, given any particular ordering of user signals. The ordering of users achieving the highest asymptotic conditional efficiency at each stage of successive detection is derived. Performance bounds based on the signal constellation structure are derived to quantify the gain of the MACE-JSIC detector compared to the MACE-SIC detector.
\end{abstract}

\section{INTRODUCTION}

The goal of multi-user detection is to correctly demodulate the information bits of mutually interfering users in a multiple-access communication system. A performance bound for joint detection is given by the maximum likelihood (ML) detector, which determines the most likely bits sent over the channel. While the ML detector achieves the lowest probability of error for joint detection, it has a complexity that is exponential in the number of users. The problem of low complexity high performance detection has been extensively studied in the past two decades, with numerous approaches proposed in the literature [1]. These include linear detectors, such as the matched filter or conventional detector [1], decorrelating detector [2], and linear minimum mean square error detector $[3,4]$, which simultaneously demodulate 
all user bits by applying a linear transformation to the received signal. Another popular approach is successive interference cancellation (SIC) in which the interfering signal of a particular user is cancelled out after making a decision on that user's bit. Many variants of interference cancellation exist in the literature, such as multi-stage interference cancellation [5-10], parallel interference cancellation (PIC) [11-13], and parallel arbitrated successive interference cancellation (PASIC) [14], among others. Most interference cancellation techniques use a linear detector as a kernel to decide on or update a user's bit before canceling its contribution to the received signal. Interference cancellation is attractive because it is a low-complexity solution that works well in many practical multi-access systems, particularly when the number of users is less than the number of dimensions of the signal space, i.e., for under-loaded communication systems.

Tutorial articles on multi-user detection are available with extensive reference lists $[8,15,16]$. Among the many suboptimal lower complexity alternatives to maximum-likelihood detection, the sequential decoding algorithm [17], cyclic decision feedback sequence detection [18], group detection [19-23] and detection based on interference cancellation from tentative decisions [12,24-27] deserve mention. An extensive literature on multi-stage detection can be found in [11,28-32] and the references therein.

In this work, we introduce a joint successive interference cancellation technique (JSIC) that gives improved performance over that of conventional successive interference cancellation (SIC). The key idea behind JSIC is to exploit the structural properties of the sub-constellation formed by the signals of two consecutive users in an ordered set to gain improvement in detector performance. The asymptotic multiuser efficiency [1], which measures the rate of decay of the bit error rate (BER) in the high SNR regime, has been used as a benchmark with which to compare the performance of detection methods. We introduce asymptotic conditional multi-user efficiency as a quantitative measure for comparing the performance of multi-user detectors that specifically employ successive interference cancellation. The optimal linear detector, in terms of achieving the highest asymptotic efficiency among all linear detectors, was proposed by Lupas and Verdu [2]. We provide an interpretation to maximal asymptotic efficiency linear detection in terms of the constellation structure and use this insight to derive maximal asymptotic conditional efficiency successive detection. For a given ordering of user signals, we derive the maximal asymptotic conditional efficiency SIC detector that achieves the maximum asymptotic conditional efficiency at each stage of successive detection among all possible SIC detectors. We also derive the optimal ordering of user signals that maximizes the asymptotic conditional efficiency at each stage of successive detection. We extend the concept of maximal asymptotic conditional efficiency (MACE) detection to the case of joint successive interference cancellation (JSIC), where at each stage of successive detection, the corresponding symbol is detected, taking into account the interference of the next user's signal in an ordered set of users. Both MACE detection algorithms proposed are robust against strong correlation of user signals, e.g., in a multi-user system where the user signals are linearly dependent. Simulation results demonstrate that 
the maximum asymptotic conditional efficiency approach significantly improves detector performance, particularly at high SNR. The focus in this paper is on understanding and mitigating the driving effects of multi-user interference (MUI) in MUI-dominated environments. As such, we consider the effects on bit-error-rate in the limit of high SNR to capture the driving factors from MUI on performance. The contributions in this paper are six-fold:

(i) introduce joint successive interference cancellation as a method of combining the joint information between consecutive users in an ordered set to attain higher performance,

(ii) formulate the maximal asymptotic linear detection problem in terms of the constellation structure,

(iii) introduce asymptotic conditional efficiency as a measure of successive interference cancellation (SIC) performance,

(iv) quantify what can be regarded as a "good ordering" of users, i.e., specifically derive the optimal ordering of users that maximizes the asymptotic conditional efficiency at each stage of successive detection,

(v) derive the successive interference canceller that optimizes the asymptotic conditional efficiency at any given stage of detection for a random ordering, and

(vi) derive performance bounds to quantify the trade-off between design complexity as well as detection complexity and performance of maximal asymptotic conditional efficiency successive detection.

The proposed techniques are most useful in stable multi-user systems with well-known parameters, such as in satellite wireless communications. Statistical models for fading channels are extensively treated in [33-37], among others. A rich literature exploring the problem of mitigating intersymbol interference introduced by fading channels include channel estimation techniques such as [38-41] and references therein, as well as techniques for mitigation of frequency-flat, frequency-selective fading [42,43], adaptive equalization of multipath [44], among others. We have assumed that a reliable channel estimation or equalization algorithm, such as the decision-feedback equalizer (DFE) ( $[33,45]$, and references therein), or the sage algorithm [46], is used to nullify the effect of any inter-symbol interference and the CDMA model is assumed to be synchronous. It has been shown [1, p. 25], that an asynchronous multi-user system can be treated as a synchronous system with a higher number of users.

The organization of the paper is as follows. The system model and list of commonly used notation are given in Section II. Section III introduces the basic idea of joint successive interference cancellation. Section IV reformulates the maximal asymptotic efficiency linear detection problem in terms of the constellation structure. Section V introduces asymptotic conditional efficiency as a measure of SIC detector performance, and the maximal asymptotic conditional efficiency SIC and JSIC detectors are introduced in Sections V and VI respectively. Section V-A derives the optimal ordering that maximizes the asymptotic conditional efficiency at each stage of SIC detection. The trade-off between complexity and performance gain is specifically treated in Section VII. Finally simulation results are presented in 
Section VIII.

\section{System Model And Notation}

We assume a synchronous BPSK-signaling CDMA model, where $\mathcal{S}=\left\{s_{i}(t)\right\}_{i=1}^{K}$ represents the set of $K$ user signals, $\left\{b_{i}\right\}_{i=1}^{K} \in(-1,+1)^{K}$ are the user symbols. The equivalent baseband received signal $y(t)$ given as

$$
y(t)=\sum_{i=1}^{K} b_{i} s_{i}(t)+n(t),
$$

where $n(t)$ is a stationary white Gaussian noise process of noise power $\sigma_{w}^{2}$. It is assumed that the number of users as well as the user signals are precisely known, as is the case in stable multi-user systems such as satellite communication systems. The user signals in $\mathcal{S}$ can be equivalently represented as vectors $\left\{\mathbf{s}_{\mathbf{i}}\right\}_{i=1}^{K}$ in a vector space, generated by an orthonormal basis $\Psi=\left\{\psi_{i}(t)\right\}_{i=1}^{N}$ that spans $\mathcal{S}$. Wherever applicable in the sequel, $\langle\cdot, \cdot\rangle$ represents the standard Euclidean inner product and $\|\cdot\|$ represents the standard $L_{2}$ norm. Thus $s_{i j}=\left\langle s_{i}(t), \psi_{j}(t)\right\rangle=\int_{-\infty}^{\infty} s_{i}(t) \psi_{j}(t) d t$ and $\mathbf{s}_{i}=\left[s_{i 1}, \ldots, s_{i N}\right]^{T}$. The received signal can be written as a vector $\mathbf{y}=\left[\left\langle y(t), \psi_{1}(t)\right\rangle, \ldots,\left\langle y(t), \psi_{N}(t)\right\rangle\right]$ in signal space, i.e.,

$$
\mathbf{y}=\mathbf{S b}+\mathbf{n},
$$

where $\mathbf{S}=\left[\mathbf{s}_{\mathbf{1}} \mathbf{s}_{\mathbf{2}} \ldots \mathbf{s}_{\mathbf{K}}\right]$ is the matrix of signal vectors, $\mathbf{b}=\left[b_{1} b_{2} \ldots b_{K}\right]$ is the vector of user symbols and $\mathbf{n} \sim \mathcal{N}\left(\mathbf{0}, \sigma^{2} \mathbf{I}\right)$ is a white Gaussian random noise vector. The energy of each user's signal is given as $A_{k}^{2}=\mathbf{s}_{k}^{T} \mathbf{s}_{k}$, where $A_{k}$ is often referred to as the amplitude of the $k^{\text {th }}$ user's signal. Another CDMA model similar to (2) and used in the literature assumes the signal space generated by the basis set $\mathcal{S}$, which need not be linearly independent. An equivalent CDMA model using $\mathcal{S}$ instead of $\Psi$ as the basis is given by

$$
\tilde{\mathbf{y}}=\mathbf{H b}+\tilde{\mathbf{n}},
$$

where $\mathbf{H}=\mathbf{S}^{\mathbf{T}} \mathbf{S}$ is the autocorrelation matrix of the user signals in $\mathcal{S}, \tilde{\mathbf{y}}=\mathbf{S}^{\mathbf{T}} \mathbf{y}$ and $\tilde{\mathbf{n}}=\mathbf{S}^{\mathbf{T}} \mathbf{n}$.

Wherever applicable, we shall use the following notation. Additional notation will be introduced as needed in the sequel.

- $\mathbf{S}(\mathbf{b})$ denotes the constellation point corresponding to the bit vector $\mathbf{b}=\left[b_{1}, b_{2}, \ldots, b_{K}\right]$.

- $P(\mathbf{v})$ denotes the point corresponding to the vector $\mathbf{v}$ in signal space. Unless stated otherwise, any vector $\mathbf{v}$ is assumed to be centered at the origin with the tip at $P(\mathbf{v})$.

- $\hat{b}_{i}$ denotes an estimate of the bit $b_{i}$.

- $\|\cdot\|$ denotes the $L_{2}$ norm.

- $\langle\cdot, \cdot\rangle$ denotes the inner product in an appropriate Hilbert space.

- $\mathcal{C}\left(\mathbf{s}_{j}\right)$ denotes the sub-constellation formed by $\sum_{i=1, i \neq j}^{K} b_{i} \mathbf{s}_{i}, b_{i}= \pm 1$.

- $\operatorname{sign}\left(b_{i}\right)$ denotes the $\operatorname{sign}\left(+\right.$ or - ) of $b_{i}$, i.e., $\operatorname{sign}\left(b_{i}\right)=+$ if $b_{i}=+1$, and $\operatorname{sign}\left(b_{i}\right)=-$ if $b_{i}=-1$. 
- For a set of user signals $\tilde{\mathcal{S}}=\left\{\mathbf{s}_{1}, \mathbf{s}_{2}, \ldots, \mathbf{s}_{k-1}, \mathbf{s}_{k}, \mathbf{s}_{k+1}, \ldots, \mathbf{s}_{K}\right\}$, the notation $\tilde{\mathcal{S}}-\mathbf{s}_{k}$ denotes the set $\left\{\mathbf{s}_{1}, \mathbf{s}_{2}, \ldots, \mathbf{s}_{k-1}, \mathbf{s}_{k+1}, \ldots, \mathbf{s}_{K}\right\}$, i.e., the set $\tilde{\mathcal{S}}$ without the user signal $\mathbf{s}_{k}$.

- $\mathcal{C}\left(\mathbf{s}_{i}^{\operatorname{sign}\left(b_{i}\right)}\right)$ denotes the sub-constellation formed by $b_{i} \mathbf{s}_{i}+\sum_{k=1, k \neq i}^{K} b_{k} \mathbf{s}_{k}$, e.g., $\mathcal{C}\left(\mathbf{s}_{i}^{+}\right)$denotes the sub-constellation $\mathbf{s}_{i}+\sum_{k=1, k \neq i}^{K} b_{k} \mathbf{s}_{k}$.

- $\mathcal{C}\left(\mathbf{s}_{i} \operatorname{sign}\left(b_{i}\right) \mathbf{s}_{j} \operatorname{sign}\left(b_{j}\right)\right)$ denotes the subconstellation formed by $b_{i} \mathbf{s}_{i}+b_{j} \mathbf{s}_{j}+\sum_{k=1, k \neq i, j}^{K} b_{k} \mathbf{s}_{k}$, e.g., $\mathcal{C}\left(\mathbf{s}_{i}^{+} \mathbf{s}_{j}^{+}\right)$denotes the subconstellation $\mathbf{s}_{i}+\mathbf{s}_{j}+\sum_{k=1, k \neq i, j}^{K} b_{k} \mathbf{s}_{k}$.

- For a given ordering $\mathcal{O}=\left\{\mathbf{s}_{i}\right\}_{i=1}^{K}$ of the user signals, $\mathcal{C}_{\mathcal{O}}\left(\mathbf{s}_{i}^{\operatorname{sign}\left(b_{i}\right)}, \mathbf{s}_{i+1}^{\operatorname{sign}\left(b_{i+1}\right)}\right)$ denotes the subconstellation corresponding to all bit vectors with the $i^{\text {th }}$ bit set to $b_{i}$, the $(i+1)^{\text {th }}$ bit set to $b_{i+1}$, and bits $1, \ldots, i-1$ set to their estimates $\{\hat{b}\}_{i=1}^{i-1}$ at the $i^{\text {th }}$ stage of detection. For example if $\mathcal{O}=\left\{\mathbf{s}_{1}, \mathbf{s}_{2}, \mathbf{s}_{3}, \mathbf{s}_{4}\right\}, \mathcal{C}_{\mathcal{O}}\left(\mathbf{s}_{2}^{+}, \mathbf{s}_{3}^{+}\right)$denotes the subconstellation $\hat{b}_{1} \mathbf{s}_{1}+\mathbf{s}_{2}+\mathbf{s}_{3}+b_{4} \mathbf{s}_{4}, b_{4}= \pm 1, \hat{b}_{1}$ being the estimate of $b_{1}$ at the $1^{\text {st }}$ stage of successive detection.

- For any ordering $\mathcal{O}=\left\{\mathbf{s}_{i}\right\}_{i=1}^{K}$ of user signals and some index set $\mathcal{I} \in\{1,2, \ldots, K\}$, let $\mathcal{S}_{I}=\left\{\mathbf{s}_{i}\right\}_{i \in \mathcal{I}}$ be some subset of user signals. Then $\mathcal{C}\left(\left\{\mathbf{s}_{i}^{\operatorname{sign}\left(b_{i}\right)}\right\}_{i \in \mathcal{I}}\right)$, denotes the sub-constellation formed by $\sum_{i \in \mathcal{I}} b_{i} \mathbf{s}_{i}+\sum_{\substack{i \notin \mathcal{I} \\ b_{i}= \pm 1}} b_{i} \mathbf{s}_{i}$, e.g., $\mathcal{C}\left(\mathbf{s}_{1}^{-}, \mathbf{s}_{2}^{+}, \mathbf{s}_{3}^{+}\right)$denotes the $2^{K-3}$ point sub-constellation $-\mathbf{s}_{1}+\mathbf{s}_{2}+$ $\mathbf{s}_{3}+\sum_{\substack{k=4 \\ b_{k}= \pm 1}}^{K} b_{k} \mathbf{s}_{k}$.

- $C H(\mathcal{C})$ denotes the convex hull of the constellation $\mathcal{C}$.

- $d(\mathbf{p}, C H(\cdot))$ denotes the minimum Euclidean distance from the vector $\mathbf{p}$ to $C H(\cdot)$.

- $d(L, C H(\cdot))$ denotes the minimum Euclidean distance from the hyperplane $L$ to $C H(\cdot)$.

\section{JOINT SUCCESSIVE INTERFERENCE CANCELLATION}

In this section we present joint successive interference cancellation in which the structural information of the sub-constellation formed by two consecutive users in an ordered set is exploited to improve the performance of conventional successive detection. The motivation behind this approach is to account for the effect of the "closest" interferer while detecting the bit of a particular user in each stage of successive detection. We first introduce the two-user joint successive interference canceller (JSIC) and then extend it to the $K$-user case.

\section{A. The two-user JSIC detector}

In this section, we describe a decision-driven detector that yields maximum-likelihood (ML) decisions for the two-user case. Figure 1 shows a two-user signal constellation formed by the user signals $\left\{\mathbf{s}_{i}, \mathbf{s}_{j}\right\}$ along with the maximum-likelihood decision regions. The two-user JSIC detector exploits the geometry of the constellation to give the maximum-likelihood solution. If $\rho=\left\langle\mathbf{s}_{i}, \mathbf{s}_{j}\right\rangle$ is the inner product between the user signals $\mathbf{s}_{i}$ and $\mathbf{s}_{j}, \overrightarrow{O B}$ represents the signal $\mathbf{p}=\mathbf{s}_{i}-\operatorname{sgn}(\rho) \mathbf{s}_{j}$. Note that the four-point constellation generated by the two-user system $\left\{\mathbf{s}_{i}, \mathbf{s}_{j}\right\}$ forms a parallelogram as shown in Figure 1. The four points $A(--), B(+-), C(++)$ and $D(-+)$ correspond to the constellation points 


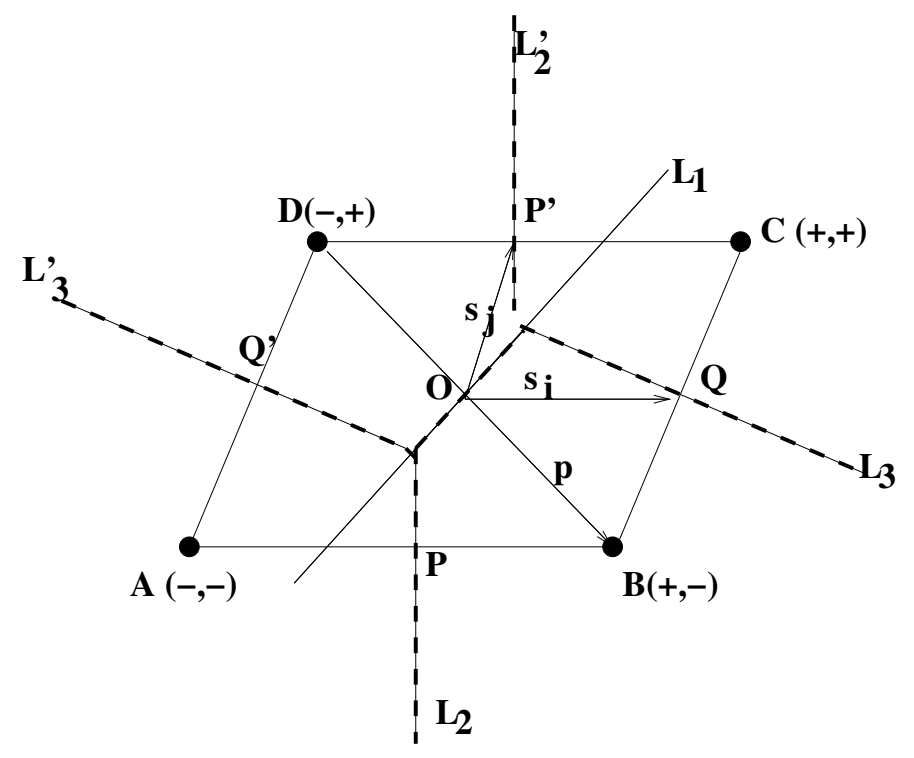

Fig. 1. ML Decision regions for two users with signal vectors $\mathbf{s}_{i}$ and $\mathbf{s}_{j}$ and $\operatorname{sgn}(\rho)>0$

$b_{i}, b_{j}=(-1,-1),(+1,-1),(+1,+1),(-1,+1)$ respectively. The ML decision regions are formed by the perpendicular bisectors of the four sides of the parallelogram $A B C D$ and $L_{1}$, the perpendicular bisector of $D B$, the shorter diagonal. The proof that $D B$ is indeed the shorter diagonal is given in [47]. The vector aligned with the shorter diagonal, i.e., the vector $\overrightarrow{O B}$ can be written $\mathbf{p}=\mathbf{s}_{i}-\operatorname{sgn}(\rho) \mathbf{s}_{j}$, in terms of the user signals. The two-user JSIC detector estimates $b_{i}$ from the received signal vector $\mathbf{y}$ using three inner product operations as shown in Table I. Figure 2 demonstrates how the two-user JSIC detector reaches a decision given a particular received vector $\mathbf{y}$. The point $P(\mathbf{y})$ representing $\mathbf{y}$ in signal space is shown as an unfilled circle at the tip of the vector $\mathbf{y}$. The darkened hyperplanes $L_{1}, L_{2}$ and $L_{3}$, labeled as steps in the algorithm represent the inner products taken in order wof precedence to reach the final bit vector estimate, $(+,+)$, for the given received vector $\mathbf{y}$.

\section{B. The K-user JSIC detector}

Consider a $K$-user system in which $\mathcal{S}=\left\{\mathbf{s}_{i}\right\}_{i=1}^{K}$ is an ordered set of user signals, arranged according to some appropriate criterion. For example, one popular approach is to order the users in a decreasing sequence of received powers. In the sequel we will also discuss an appropriate ordering of users in terms of multi-user efficiency. The extension of the two-user JSIC algorithm to the $K$-user case is given in Table II. The $K$-user JSIC detector estimates $\mathbf{b}$ from the received signal y in $2(K-1)+1$ steps.

It is easy to verify that the JSIC detector performs $2(K-1)+1$ inner product operations to estimate the bit vector $\mathbf{b}$, and therefore has computational complexity $\mathcal{O}(K)$, i.e., linear in the number of users. 
TABLE I

The two-user JSIC detector

Step 1: Decide to which side of $L_{1}$ the received point $\mathbf{y}$ lies:
\[ \hat{b}=\operatorname{sgn}(\langle\mathbf{y}, \mathbf{p}\rangle), \]

Step 2: Re-center the constellation at the point $P$ if $\hat{b}>0$ (or $P^{\prime}$ if $\hat{b}<0$ ) and decide on which side of the line $L_{2}\left(\right.$ or $\left.L_{2}^{\prime}\right)$ the received point lies:

$$
\hat{b}_{i}=\operatorname{sgn}\left(\left\langle\left(\mathbf{y}+\hat{b} \operatorname{sgn}(\rho) \mathbf{s}_{j}\right), \mathbf{s}_{i}\right\rangle\right)
$$

Step 3: Then $b_{j}$ is estimated as:

$$
\hat{b}_{j}=\operatorname{sgn}\left(\left\langle\left(\mathbf{y}-\hat{b}_{i} \mathbf{s}_{i}\right), \mathbf{s}_{j}\right\rangle\right)
$$

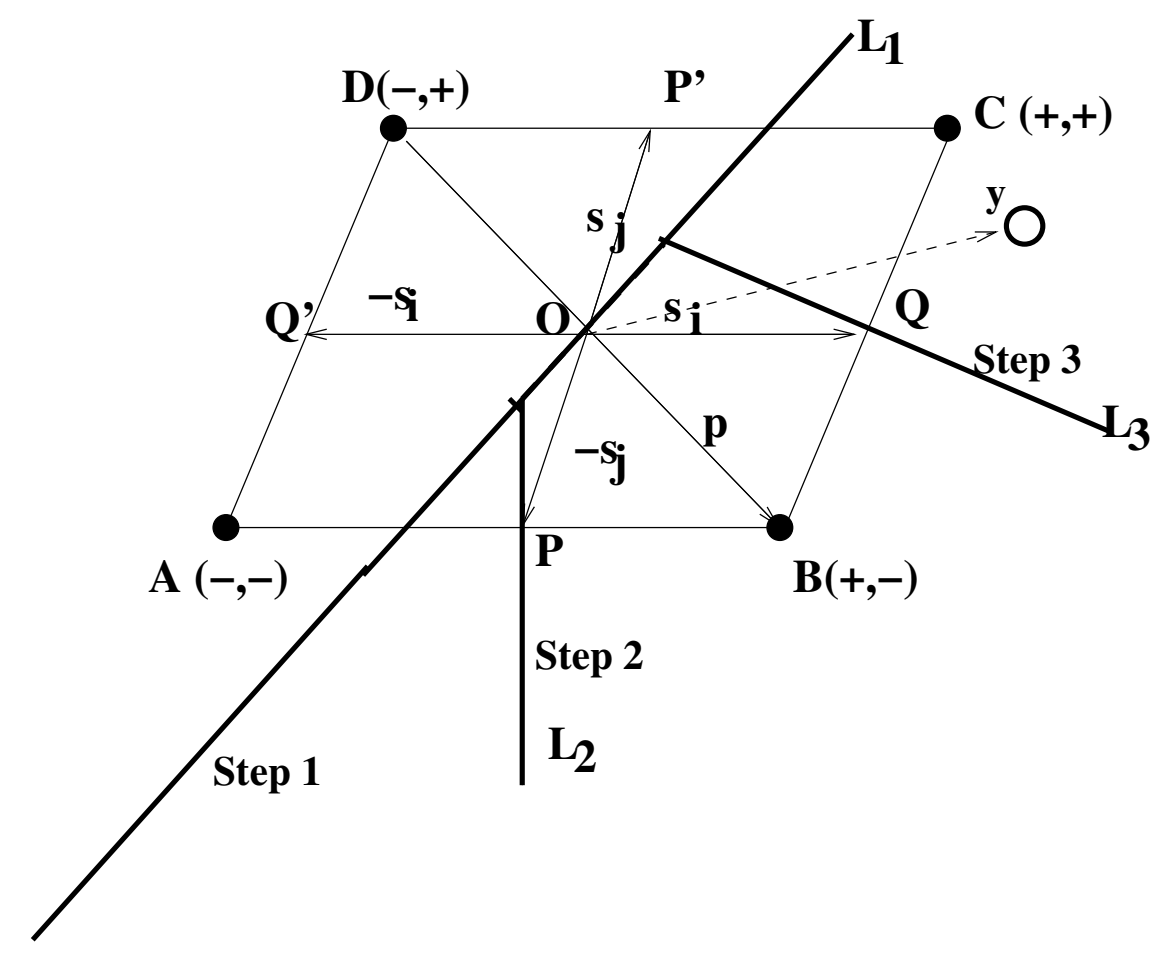

Fig. 2. Illustration of the two-user JSIC algorithm for a given received vector.

The key idea behind the JSIC detector is to account for a significant interferer in an ordered set using a two-user locally ML kernel. As with all SIC detectors, the performance of the JSIC detector will be a function of the ordering of the users in the algorithm. Improved estimates might be obtained for each user at each stage of successive detection through a different (more favorable) ordering. Figure 3 demonstrates how the $K$-user JSIC algorithm works, step by step, for a given a received vector. Similar to 2 , the darkened hyperplanes represent the inner products labeled in order of precedence to reach the final bit vector estimate, in this case, $(+,-,+)$. The received vector $\mathbf{y}$ is given by the vector pointing 
TABLE II

The $K$-user JSIC detector

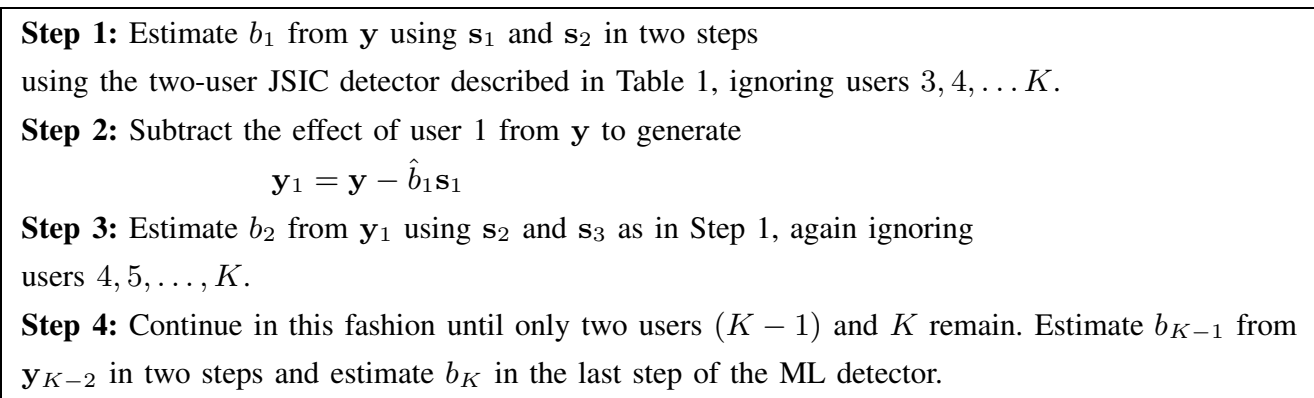

to the unfilled circle, which represents the received point $P(\mathbf{y})$ in signal space.

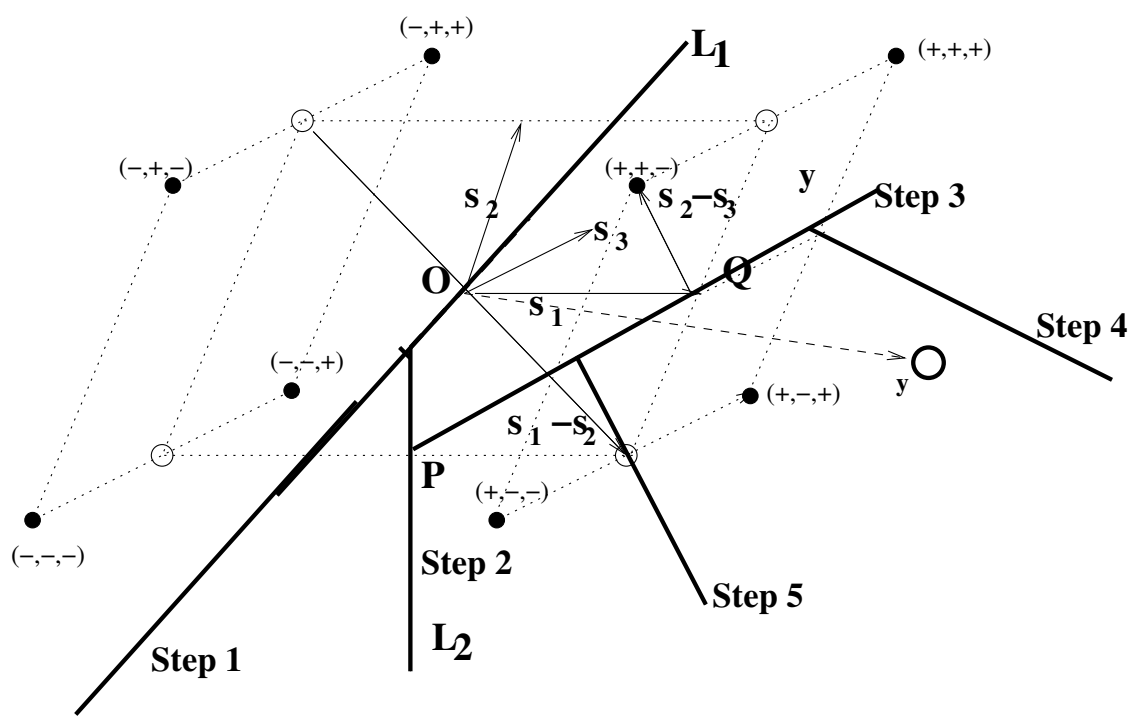

Fig. 3. Illustration of the $K$-user JSIC algorithm for a given received vector.

\section{MAXIMAL ASYMPTOTIC EFFICIENCY LINEAR DETECTOR: A STRUCTURAL PERSPECTIVE}

In this section, we formulate maximal asymptotic efficiency linear detection in terms of the structural geometry of the multi-user signal constellation, and use this perspective in subsequent sections to develop SIC and JSIC detectors optimized in terms of the slope of the conditional probability of error. To evaluate the performance of the detectors proposed in this work, we focus on the asymptotic multi-user efficiency of the multi-user detector. Asymptotic multi-user efficiency was first proposed by Lupas and Verdu [2] and is a quantitative measure of detector performance in terms of its bit error rate (BER) at high SNR. 
The bit error rate of the $k^{\text {th }}$ user is denoted $P_{k}(\sigma)$ and is a function of all the user signals interfering in the channel, the multi-user detector used and the noise variance, $\sigma^{2}$. The multi-user efficiency of a detector for the $k^{\text {th }}$ user in a multi-user detector, denoted $\eta_{k}(\sigma)$, is given as the ratio of the effective energy, $e_{k}(\sigma)$, that the $k^{\text {th }}$ user would require in order to achieve the same bit error rate $P_{k}(\sigma)$ in a single-user channel, to the actual energy of the $k^{\text {th }}$ user, $A_{k}^{2}$, in the multi-user channel, i.e.,

$$
\eta_{k}(\sigma)=\frac{e_{k}(\sigma)}{A_{k}^{2}}
$$

where $P_{k}(\sigma)=Q\left(\sqrt{e_{k}(\sigma)} / \sigma\right)$ and $A_{k}$ is the amplitude of the $k^{\text {th }}$ user.

In the absence of interfering users, the effective energy will be the same as the actual energy. Because of multi-user interference, the bit error rate is increased, and hence the effective energy is less than the actual energy of the $k^{\text {th }}$ user. The multi-user efficiency $\eta_{k}(\sigma)$ is therefore always less than unity. We are interested in the asymptotic behavior of $\eta_{k}(\sigma)$ and hence the effect of multi-user interference as the noise variance vanishes. Mathematically, the asymptotic multi-user efficiency of a detector for the $k^{\text {th }}$ user can be calculated to be [1]

$$
\eta_{k}=\frac{2}{A_{k}^{2}} \lim _{\sigma \rightarrow 0} \sigma^{2} \log \frac{1}{P_{k}(\sigma)} .
$$

We state without proof the following well-known results [1, p. 352].

Theorem 4.1: Let $P\left(\mathbf{b} \rightarrow \mathbf{b}^{\prime}\right)$ denote the probability that the multi-user detector outputs $\mathbf{b}^{\prime}$ given b was transmitted. Let us denote the minimum distance from the constellation point $S(\mathbf{b})$ to $D\left(\mathbf{b}^{\prime}\right)$ (decision region corresponding to $S\left(\mathbf{b}^{\prime}\right)$ ) by $\Delta\left(\mathbf{b}, \mathbf{b}^{\prime}\right)=\min _{v \in D\left(\mathbf{b}^{\prime}\right)}\|v-S(\mathbf{b})\|$. If $D\left(\mathbf{b}^{\prime}\right)$ is a convex polytope that includes its boundary and does not depend on $\sigma$, then

$$
\lim _{\sigma \rightarrow 0} 2 \sigma^{2} \log \frac{1}{P\left(\mathbf{b} \rightarrow \mathbf{b}^{\prime}\right)}=\Delta^{2}\left(\mathbf{b}, \mathbf{b}^{\prime}\right) .
$$

For completeness we include this extension here, though the result parallels other well known results from large deviations theory [48], [49, Theorem 5.2, p. 77].

Theorem 4.2: Let the decision region $D\left(\mathbf{b}^{\prime}\right)$ of $S\left(\mathbf{b}^{\prime}\right)$ be the union of convex polytopes $\left\{D_{i}(\mathbf{b})\right\}_{i=1}^{L}$. Let $\Delta_{i}\left(\mathbf{b}, \mathbf{b}^{\prime}\right)=\min _{v \in D_{i}\left(\mathbf{b}^{\prime}\right)}\|v-S(\mathbf{b})\| i=1,2, \ldots, L$. Then

$$
\lim _{\sigma \rightarrow 0} 2 \sigma^{2} \log \frac{1}{P\left(\mathbf{b} \rightarrow \mathbf{b}^{\prime}\right)}=\min \left\{\Delta_{i}^{2}\left(\mathbf{b}, \mathbf{b}^{\prime}\right)\right\}_{i=1}^{L}
$$

It follows from Theorem 4.2 that for detectors with convex decision regions, or decision regions that comprise a union of convex sets, the asymptotic multi-user efficiency of the detector for the $k^{\text {th }}$ user is given by the shortest distance squared from a constellation point to the decision boundary separating it from another point that differs in the $k^{\text {th }}$ bit, normalized by $A_{k}^{2}$. This can be written,

$$
\eta_{k}=\min _{\mathbf{b}, \mathbf{b}^{\prime} \in\{-1,+1\}^{K}, b_{k} \neq b_{k}^{\prime}} \frac{\Delta^{2}\left(\mathbf{b}, \mathbf{b}^{\prime}\right)}{A_{k}^{2}}
$$

This has a number of implications for optimizing the performance of the JSIC detector that we will explore in the sequel. 
The "best" (or optimal) linear detector introduced by Lupas and Verdu in [2] achieves the highest asymptotic multi-user efficiency among all linear detectors. By a linear detector, we mean any detector that outputs bit estimates as $\hat{b}_{k}=\operatorname{sgn}\left(\left\langle\mathbf{y}, \mathbf{v}_{k}\right\rangle\right), k=1,2, \ldots, K$, for some vector $\mathbf{v}_{k}$. The asymptotic efficiency of the optimal linear detector for the $k^{\text {th }}$ user is given in [2] as

$$
\eta_{k}=\max _{\mathbf{w} \in \Re^{K}} \frac{1}{\mathbf{w}^{T} \mathbf{H w}}\left(\mathbf{h}_{k}^{T} \mathbf{w}-\sum_{j \neq k}\left|\mathbf{h}_{j}^{T} \mathbf{w}\right|\right)^{2},
$$

where the matrix $\mathbf{H}$ is as defined in the CDMA model from (3), and $\mathbf{H}=\left[\mathbf{h}_{1} \mathbf{h}_{2} \ldots \mathbf{h}_{K}\right]$.

Note that, in general, the best linear detector must be found by solving the convex optimization (8) over all linear detectors $\mathbf{w}$. Using (2), when the signal space is generated by an orthonormal basis $\Psi=$ $\left\{\psi_{1}(t), \psi_{2}(t), \ldots, \psi_{N}(t)\right\}$, if $\mathbf{v}$ is a vector represented with $\Psi$, and $\mathbf{w}$ is the corresponding representation with respect to $\mathcal{S}$, then $\mathbf{v}=\mathbf{S w}$. Therefore, $\|\mathbf{v}\|^{2}=\mathbf{w}^{T} \mathbf{H w}$. Therefore, in the model (2), the maximum asymptotic efficiency of a linear detector for the $k^{\text {th }}$ user is given by

$$
\eta_{k}=\max _{\mathbf{v}_{k} \in \Re^{K}} \frac{1}{\mathbf{v}_{k}^{T} \mathbf{v}_{k}}\left(\mathbf{s}_{k}^{T} \mathbf{v}_{k}-\sum_{j \neq k}\left|\mathbf{s}_{j}^{T} \mathbf{v}_{k}\right|\right)^{2}
$$

In this work, we interpret the above convex optimization problem in terms of the structure of the constellation. We analyze the detector given in [2] in terms of the constellation structure and apply this perspective to extend the idea of maximal asymptotic efficiency multi-user detection to successive interference cancellation and derive an appropriate ordering of users for successive detection.

Theorem 4.3: For a linear system represented as in (2), the maximum asymptotic efficiency of a detector for the $k^{\text {th }}$ user, denoted $\eta_{k \text {,max }}$, achievable among all possible linear detectors outputting the decision of the $k^{\text {th }}$ bit as $\hat{b}_{k}=\operatorname{sgn}\left(\left\langle\mathbf{y}, \mathbf{v}_{k}\right\rangle\right), \mathbf{v}_{k} \in \Re^{N}$, is equal to the minimum distance squared from the origin to the convex hull of the sub-constellation with the $k^{\text {th }}$ user's bit set to \pm 1 , i.e., $\mathcal{C}\left(\mathbf{s}_{k}^{ \pm}\right)$, normalized by the energy of the $k^{\text {th }}$ user, i.e.,

$$
\eta_{k, \max }=\frac{d^{2}\left(\mathbf{0}, C H\left(\mathcal{C}\left(\mathbf{s}_{k}^{ \pm}\right)\right)\right)}{A_{k}^{2}}
$$

where $d^{2}\left(\mathbf{0}, C H\left(\mathcal{C}\left(\mathbf{s}_{k}^{ \pm}\right)\right)\right)$is the minimum distance squared from the origin to the convex hull of the sub-constellation $\mathcal{C}\left(\mathbf{s}_{k}^{ \pm}\right)$, and $A_{k}$ is the amplitude of the $k^{\text {th }}$ user. The maximum asymptotic efficiency linear detector will output bit estimates

$$
\hat{b}_{k}=\operatorname{sgn}\left(\left\langle\mathbf{v}_{k, \mathrm{opt}}, \mathbf{y}\right\rangle\right)
$$

where $\mathbf{v}_{k \text {,opt }}$ is proportional to the minimum distance vector $\mathbf{v}^{*}$ from the origin to $C H\left(\mathcal{C}\left(\mathbf{s}_{k}^{ \pm}\right)\right)$, such that $\left\|\mathbf{v}^{*}\right\|^{2}=d^{2}\left(\mathbf{0}, C H\left(\mathcal{C}\left(\mathbf{s}_{k}^{ \pm}\right)\right)\right)$.

Proof: For notational simplicity, we will denote $\mathcal{C}\left(\mathbf{s}_{k}^{+}\right)$and $\mathcal{C}\left(\mathbf{s}_{k}^{-}\right)$as $\mathcal{C}_{1}$ and $\mathcal{C}_{2}$ respectively. Also, for clarity, we denote $P(\mathbf{v})$ as the point corresponding to the vector $\mathbf{v}$. The asymptotic efficiency of a 
detector with probability of error $P_{k}(\sigma)$ for the $k^{\text {th }}$ user, $\eta_{k}$, is given by [1]:

$$
\eta_{k}=\frac{1}{A_{k}^{2}} \lim _{\sigma \rightarrow 0} 2 \sigma^{2} \log \frac{1}{P_{k}(\sigma)} .
$$

With BPSK signaling, any pair of constellation points $\mathbf{S}(\mathbf{b})$ and $\mathbf{S}(\overline{\mathbf{b}}), \mathbf{b}$ and $\overline{\mathbf{b}}$ being bitwise complements, will be radially symmetric about the origin 0 . Thus the sub-constellations $\mathcal{C}_{1}$ and $\mathcal{C}_{2}$ will also be radially symmetric about $\mathbf{0}$. Therefore, from symmetry considerations, we must have

$$
d^{2}\left(\mathbf{0}, C H\left(\mathcal{C}\left(\mathbf{s}_{k}^{+}\right)\right)\right)=d^{2}\left(\mathbf{0}, C H\left(\mathcal{C}\left(\mathbf{s}_{k}^{-}\right)\right)\right) .
$$

We must also have that the conditional probability of error given $b_{k}=1$, denoted $P_{k}\left(\sigma \mid b_{k}=+1\right)$, is equal to the conditional probability of error given $b_{k}=-1, P_{k}\left(\sigma \mid b_{k}=-1\right)$. We denote $\operatorname{Prob}\left(b_{k}=i\right), i= \pm 1$ as the probability that $b_{k}= \pm 1$ was sent and assume that $\operatorname{Prob}\left(b_{k}=i\right)=\frac{1}{2}, i= \pm 1$. The bit-error-rate (BER), $P_{k}(\sigma)$, of a given linear detector that estimates the $k^{\text {th }}$ bit as $\hat{b}_{k}=\operatorname{sgn}\left(\left\langle\mathbf{y}, \mathbf{v}_{k}\right\rangle\right)$ is given by

$$
\begin{aligned}
P_{k}(\sigma) & =P_{k}\left(\sigma \mid b_{k}=+1\right) \operatorname{Prob}\left(b_{k}=+1\right)+P_{k}\left(\sigma \mid b_{k}=-1\right) \operatorname{Prob}\left(b_{k}=-1\right) \\
& =\frac{1}{2} P_{k}\left(\sigma \mid b_{k}=+1\right)+\frac{1}{2} P_{k}\left(\sigma \mid b_{k}=-1\right) \\
& =P_{k}\left(\sigma \mid b_{k}=+1\right) \\
& =\frac{1}{2^{K-1}} \sum_{\left\{b_{i}\right\}_{i=1}^{K}, i \neq k, b_{i}= \pm 1} \operatorname{Prob}\left(\hat{b}_{k} \neq b_{k} \mid b_{1}, \ldots, b_{k-1}, b_{k+1}, \ldots, b_{K}, b_{k}=+1\right) \\
& =\frac{1}{2^{K-1}} \sum_{\left\{b_{i}\right\}_{i=1}^{K}, i \neq k, b_{i}= \pm 1} Q\left(\frac{\left\langle\mathbf{v}_{k}, \mathbf{s}_{k}+\sum_{i \neq k} b_{i} \mathbf{s}_{i}\right\rangle}{\sigma \sqrt{\mathbf{v}_{k}^{T} \mathbf{v}_{k}}}\right) \\
& =\frac{1}{2^{K-1}} \sum_{\left\{b_{i}\right\}_{i=1}^{K}, i \neq k, b_{i}= \pm 1} Q\left(\frac{\left\langle\mathbf{v}_{k}, \mathbf{s}_{k}+\sum_{i \neq k} b_{i} \mathbf{s}_{i}\right\rangle}{\sigma\left\|\mathbf{v}_{k}\right\|}\right)
\end{aligned}
$$

where $\operatorname{Prob}\left(\hat{b}_{k} \neq b_{k} \mid b_{1}, \ldots, b_{k-1}, b_{k+1}, \ldots, b_{K}, b_{k}=+1\right)$ is the conditional probability that $\hat{b}_{k}=-1$ given $b_{k}=+1$ and given that $\left\{b_{i}\right\}_{i=1}^{K}, i \neq k$ were the other users' bits. There are $2^{K-1}$ possible combinations for the other users' bits and hence the summation has been taken over all possible configurations of the bit vector $\mathbf{b}=\left\{b_{j}\right\}_{j=1}^{K}$ such that $b_{k}=1$. For each bit vector combination $\left\{b_{j}\right\}_{j=1}^{K}, b_{k}=1$, $\operatorname{Prob}\left(\hat{b}_{k} \neq b_{k} \mid b_{1}, \ldots, b_{k-1}, b_{k+1}, \ldots, b_{K}, b_{k}=+1\right)$ will be given by $Q\left(\frac{\left\langle\mathbf{v}_{k}, \mathbf{s}_{k}+\sum_{i \neq k} b_{i} \mathbf{s}_{i}\right\rangle}{\sigma\left\|\mathbf{v}_{k}\right\|}\right)$, since this measures the probability that $\mathbf{n} \sim \mathcal{N}\left(\mathbf{0}, \sigma^{2} \mathbf{I}\right)$, is greater than $\left\langle\mathbf{v}_{k}, \mathbf{s}_{k}+\sum_{i \neq k} b_{i} \mathbf{s}_{i}\right\rangle$ in each of the $2^{K-1}$ cases that an error is made on $\hat{b}_{k}$. We index the $2^{K-1}$ possible combinations of $\left\{b_{i}\right\}_{i=1, i \neq k}^{K}$ as $j=1,2, \ldots, 2^{K-1}$ and denote the corresponding bit vector as $\mathbf{b}^{j}$ and the normalized inner product 
$\frac{\left\langle\mathbf{v}_{k}, \mathbf{s}_{k}+\sum_{i \neq k} b_{i}^{j} \mathbf{s}_{i}\right\rangle}{\left\|\mathbf{v}_{k}\right\|}$ as $\left\{\alpha_{j}\right\}_{j=1}^{2^{K-1}}$. We define

$$
\begin{aligned}
\alpha_{\min } & =\min _{j} \alpha_{j} \\
& =\min _{j} \frac{\left\langle\mathbf{v}_{k}, \mathbf{s}_{k}+\sum_{i=1, i \neq k}^{K} b_{i}^{j} \mathbf{s}_{i}\right\rangle}{\left\|\mathbf{v}_{k}\right\|} \\
& =\min _{P(\mathbf{c}) \in \mathcal{C}_{1}} \frac{\left\langle\mathbf{v}_{k}, \mathbf{c}\right\rangle}{\left\|\mathbf{v}_{k}\right\|},
\end{aligned}
$$

i.e., $\alpha_{\min }$ is the minimum normalized inner product between $\mathbf{v}_{k}$ and any constellation point belonging to the sub-constellation $\mathcal{C}_{1}$. Note that $\alpha_{\min }$ will depend on $\mathbf{v}_{k}$. From large deviation theory [48], it can be shown that [49, Section 3.2] as $\sigma \rightarrow 0, P_{k}(\sigma)$ is dominated by the term that has a $Q$-function with the minimum argument. The following derivation in the context of asymptotic efficiency for equalizers [49] is reproduced here for convenience.

The product of the asymptotic efficiency of the detector for the $k^{\text {th }}$ user and the energy of the $k^{\text {th }}$ user, sometimes referred to as the asymptotic multi-user energy, can be expressed as:

$$
\begin{aligned}
A_{k}^{2} \eta_{k} & =\lim _{\sigma \rightarrow 0} 2 \sigma^{2} \log \frac{1}{P_{k}(\sigma)} \\
& =-\lim _{\sigma \rightarrow 0} 2 \sigma^{2} \log \left(\frac{1}{2^{K-1}} \sum_{j=1}^{2^{K-1}} Q\left(\frac{\alpha_{j}}{\sigma}\right)\right) \\
& =-\lim _{\sigma \rightarrow 0} 2 \sigma^{2}\left[\log \left(\frac{1}{2^{K-1}}\right)+\log \left(\sum_{j=1}^{2^{K-1}} Q\left(\frac{\alpha_{j}}{\sigma}\right)\right)\right] \\
& =-\lim _{\sigma \rightarrow 0} 2 \sigma^{2} \log \left(\sum_{j=1}^{2^{K-1}} Q\left(\frac{\alpha_{j}}{\sigma}\right)\right) \\
& =-\lim _{\sigma \rightarrow 0} 2 \sigma^{2} \log \left[Q\left(\frac{\alpha_{\min }}{\sigma}\right)\left(1+\sum_{j=1, j \neq l: \alpha_{\min }=\alpha_{l}}^{2^{K-1}} \frac{Q_{\left(\frac{\alpha_{j}}{\sigma}\right)}}{Q\left(\frac{\alpha_{\min }}{\sigma}\right)}\right)\right] \\
& =-\lim _{\sigma \rightarrow 0} 2 \sigma^{2}\left[\log \left[Q\left(\frac{\alpha_{\min }}{\sigma}\right)\right]+\log \left[\left(1+\sum_{j=1, j \neq l: \alpha_{\min }=\alpha_{l}} \frac{Q\left(\frac{\alpha_{j}}{\sigma}\right)}{Q\left(\frac{\alpha_{\min }}{\sigma}\right)}\right)\right]\right]
\end{aligned}
$$

The last logarithm can be expanded in a Taylor series and vanishes in the limit $\sigma \rightarrow 0$. Therefore, we obtain,

$$
\begin{aligned}
A_{k}^{2} \eta_{k}^{2} & =-\lim _{\sigma \rightarrow 0} 2 \sigma^{2} \log \left(Q\left(\frac{\alpha_{\min }}{\sigma}\right)\right) \\
& =-\lim _{x \rightarrow \infty} \frac{2 \alpha_{\min }^{2}}{x^{2}} \log (Q(x))
\end{aligned}
$$

where $x=\alpha_{\min } / \sigma$. 
Using the well-known inequality [1, p. 98],

$$
\frac{1}{\sqrt{2 \pi} x}\left(1-x^{2}\right) e^{-x^{2} / 2}<Q(x)<\frac{1}{\sqrt{2 \pi} x} e^{-x^{2} / 2}, x>1,
$$

and taking the limit as $x \rightarrow \infty$ on both sides, it is easy to verify that

$$
\lim _{x \rightarrow \infty} \frac{2 \alpha_{\min }^{2}}{x^{2}} \log Q(x)=-\alpha_{\min }^{2} .
$$

Therefore,

$$
\eta_{k}=\frac{\alpha_{\min }^{2}}{A_{k}^{2}} .
$$

Thus to maximize the asymptotic efficiency, $\eta_{k}$, for the $k^{\text {th }}$ user, we maximize the minimum normalized inner product between $\mathbf{v}_{k}$ and a constellation point in $\mathcal{C}_{1}$. Denoting the vector $\mathbf{v}_{k} \in \Re^{N}$ that maximizes $\eta_{k}$ as $\mathbf{v}_{k, \text { opt }}$, we have

$$
\mathbf{v}_{k, \text { opt }}=\arg \max _{\mathbf{v}_{k} \in \Re^{N}} \min _{P(\mathbf{c}) \in \mathcal{C}_{1}} \frac{\left\langle\mathbf{v}_{k}, \mathbf{c}\right\rangle}{\left\|\mathbf{v}_{k}\right\|}
$$

Let us denote $\Delta_{k}\left(\mathbf{v}_{k}\right)=\min _{P(\mathbf{c}) \in \mathcal{C}_{1}}\left(\left\langle\mathbf{v}_{k}, \mathbf{c}\right\rangle /\left\|\mathbf{v}_{k}\right\|\right)$ and $\Delta_{k, \text { opt }}=\max _{\mathbf{v}_{k} \in \Re^{N}} \Delta_{k}\left(\mathbf{v}_{k}\right)$. Therefore, $\Delta_{k, \text { opt }}=\min _{P(\mathbf{c}) \in \mathcal{C}_{1}}\left(\left\langle\mathbf{v}_{k, \text { opt }}, \mathbf{c}\right\rangle\right) /\left\|\mathbf{v}_{k, \text { opt }}\right\|$. Let $\mathbf{v}^{*}$ be the minimum distance vector from the origin to the convex hull of the sub-constellation $\mathcal{C}_{1}$, i.e., $\mathbf{v}^{*}=\arg \min _{P(\mathbf{v}) \in C H\left(\mathcal{C}_{1}\right)}\|\mathbf{v}\|^{2}$. We now show that $\Delta_{k, \text { opt }}$ is the minimum distance from the origin to the convex hull of $\mathcal{C}_{1}$., i.e., $\Delta_{k \text {,opt }}=\left\|\mathbf{v}^{*}\right\|$. The convex hull of any set of points is the minimum convex set containing those points. Any point $\mathbf{p} \in C H\left(\mathcal{C}_{1}\right)$ is given by $\mathbf{p}=\sum_{i} \lambda_{i} \mathbf{c}_{i}, \sum_{i} \lambda_{i}=1, \mathbf{c}_{i} \in \mathcal{C}_{1}$. Before we proceed, we state the following well-known property of convex sets [50, Theorem 1, p. 69], which will be used throughout the proof.

Property 4.1: If $\mathbf{v}^{*}$ be the minimum distance vector from the origin to a convex set $\mathcal{C}$ and $P\left(\mathbf{c} \neq \mathbf{v}^{*}\right)$ is a point in $\mathcal{C}$, then $\left\langle\left(\mathbf{c}-\mathbf{v}^{*}\right), \mathbf{v}^{*}\right\rangle \geq 0$. Equality is achieved only when the point lies on the hyperplane orthogonal to $\mathbf{v}^{*}$. This is graphically illustrated in Figure 4, where, for the point $C=P(\mathbf{c})$, we have $\left\langle\left(\mathbf{c}-\mathbf{v}^{*}\right), \mathbf{v}^{*}\right\rangle>0$ and for the point $D=P(\mathbf{d})$, we have $\left\langle\left(\mathbf{d}-\mathbf{v}^{*}\right), \mathbf{v}^{*}\right\rangle=0$.

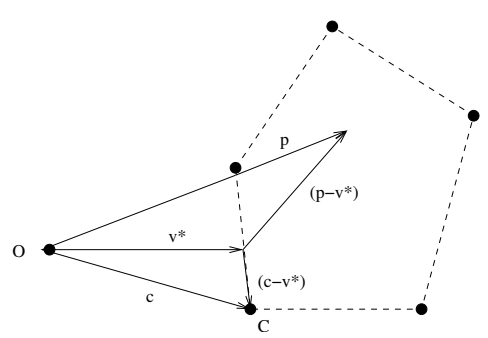

Fig. 4. Illustration of Property 4.1

Case 1: Let us first consider the case when $P\left(\mathbf{v}^{*}\right) \in \mathcal{C}_{1}$, i.e, $P\left(\mathbf{v}^{*}\right)$ itself is a constellation point in the sub-constellation $\mathcal{C}_{1}$. Since, $P\left(\mathbf{v}^{*}\right) \in \mathcal{C}_{1}$, if $\mathbf{v}_{k}$ lies along $\mathbf{v}^{*}$, i.e., $\mathbf{v}_{k}=\alpha \mathbf{v}^{*}, \alpha>0$, we must have: 


$$
\begin{aligned}
\frac{\left\langle\mathbf{v}^{*}, \mathbf{v}_{k}\right\rangle}{\left\|\mathbf{v}_{k}\right\|} & =\frac{\left\|\mathbf{v}^{*}\right\|\left\|\mathbf{v}_{k}\right\|}{\left\|\mathbf{v}_{k}\right\|} \\
& =\left\|\mathbf{v}^{*}\right\| .
\end{aligned}
$$

It follows from Property 4.1 , that for any $P(\mathbf{c}) \in \mathcal{C}_{1}, \mathbf{c} \neq \mathbf{v}^{*}$, we must have $\left\langle\mathbf{c}-\mathbf{v}^{*},-\mathbf{v}^{*}\right\rangle<0$. By construction, $\mathbf{v}^{*}$ and $\mathbf{v}_{k}$ are in the same direction. Therefore, we must have

$$
\begin{aligned}
\frac{\left\langle\mathbf{c}, \mathbf{v}_{k}\right\rangle}{\left\|\mathbf{v}_{k}\right\|} & =\frac{\left\langle\mathbf{c}-\mathbf{v}^{*}, \mathbf{v}_{k}\right\rangle}{\left\|\mathbf{v}_{k}\right\|}+\frac{\left\langle\mathbf{v}^{*}, \mathbf{v}_{k}\right\rangle}{\left\|\mathbf{v}_{k}\right\|} \\
& >\frac{\left\langle\mathbf{v}^{*}, \mathbf{v}_{k}\right\rangle}{\left\|\mathbf{v}_{k}\right\|} \\
& =\left\|\mathbf{v}^{*}\right\|
\end{aligned}
$$

since $\left\langle\mathbf{c}-\mathbf{v}^{*}, \mathbf{v}_{k}\right\rangle>0$. Therefore, if $\mathbf{v}_{k}^{\alpha}=\alpha \mathbf{v}^{*}$, for any $\alpha>0$,

$$
\Delta_{k}\left(\mathbf{v}_{k}^{\alpha}\right)=\left\|\mathbf{v}^{*}\right\| \text {. }
$$

If $\mathbf{v}_{k} \neq \alpha \mathbf{v}^{*}, \alpha>0$, then the vectors $\mathbf{v}^{*}$ and $\mathbf{v}_{k}$ will subtend some angle $\theta \neq 0$ with each other, i.e.,

$$
\begin{aligned}
\frac{\left\langle\mathbf{v}^{*}, \mathbf{v}_{k}\right\rangle}{\left\|\mathbf{v}_{k}\right\|} & =\frac{\left\|\mathbf{v}^{*}\right\|\left\|\mathbf{v}_{k}\right\| \cos \theta}{\left\|\mathbf{v}_{k}\right\|} \\
& =\left\|\mathbf{v}^{*}\right\| \cos \theta \\
& <\left\|\mathbf{v}^{*}\right\| .
\end{aligned}
$$

Therefore if $\mathbf{v}_{k} \neq \alpha \mathbf{v}^{*}, \alpha>0$, we must have

$$
\Delta_{k}\left(\mathbf{v}_{k}\right)<\left\|\mathbf{v}^{*}\right\| .
$$

From (23) and (25) we infer that $\mathbf{v}_{k \text {,opt }}$ is proportional to the minimum distance vector $\mathbf{v}^{*}$ from the origin to the convex hull of $\mathcal{C}_{1}$ and that $\Delta_{k, \text { opt }}=\left\|\mathbf{v}^{*}\right\|$.

Case 2: Now let us consider the case where $\mathbf{v}^{*}$ is not a constellation point, as shown in Figure 5. Let $P$ be the projection of the origin onto the convex set $C H\left(\mathcal{C}_{1}\right)$, i.e., $P$ is the unique point in $C H\left(\mathcal{C}_{1}\right)$ that is geometrically closest to the origin $O$. Let $L$ be the $(N-1)$-dimensional hyperplane orthogonal to the vector $\mathbf{p}=\overrightarrow{O P}$ and passing through $P$. Therefore, by construction, $\mathbf{p}=\mathbf{v}^{*}, L \perp \mathbf{p}$. For any constellation point $P(\mathbf{c}) \in L$ and any vector $\mathbf{v}_{k}=\alpha \mathbf{p}, \alpha>0$, we can express the normalized inner product between $\mathbf{c}$ and $\mathbf{v}_{k}$ as:

$$
\begin{aligned}
\frac{\left\langle\mathbf{c}, \mathbf{v}_{k}\right\rangle}{\left\|\mathbf{v}_{k}\right\|} & =\frac{\|\mathbf{c}\|\|\alpha \mathbf{p}\| \cos 90^{\circ}}{\|\alpha \mathbf{p}\|} \\
& =\frac{\|\mathbf{c}\|\|\mathbf{p}\| \cos 90^{\circ}}{\|\mathbf{p}\|} \\
& =\frac{\|\mathbf{p}\|^{2}}{\|\mathbf{p}\|} \\
& =\|\mathbf{p}\| \\
& =\left\|\mathbf{v}^{*}\right\| .
\end{aligned}
$$




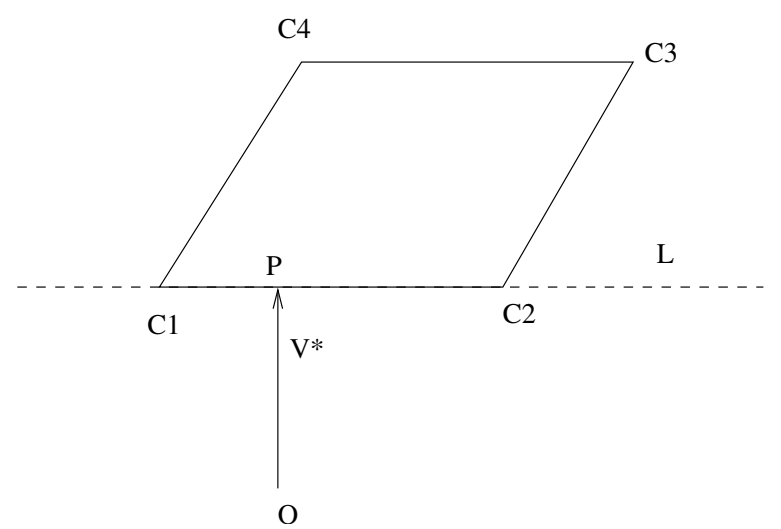

Fig. 5. Illustration of the case where the minimum distance vector is not a constellation point

Therefore for any constellation point $P(\mathbf{c}) \in \mathcal{C}_{1}-L$, i.e., $P(\mathbf{c}) \in \mathcal{C}_{1}$, but $P(\mathbf{c})$ does not lie along $L$, we must have, from Property 4.1, that $\left\langle\mathbf{c}-\mathbf{v}^{*}, \mathbf{v}^{*}\right\rangle>0$. Hence, for $\mathbf{v}_{k}=\alpha \mathbf{p}, \alpha>0$, we have

$$
\begin{aligned}
\frac{\left\langle\mathbf{c}, \mathbf{v}_{k}\right\rangle}{\left\|\mathbf{v}_{k}\right\|} & =\frac{\left\langle\mathbf{v}^{*}+\mathbf{c}-\mathbf{v}^{*}, \mathbf{v}_{k}\right\rangle}{\left\|\mathbf{v}_{k}\right\|} \\
& =\frac{\left\langle\mathbf{v}^{*}, \mathbf{v}_{k}\right\rangle}{\left\|\mathbf{v}_{k}\right\|}+\frac{\left\langle\mathbf{c}-\mathbf{v}^{*}, \mathbf{v}_{k}\right\rangle}{\left\|\mathbf{v}_{k}\right\|} \\
& =\frac{\left\|\mathbf{v}^{*}\right\| \alpha\left\|\mathbf{v}^{*}\right\|}{\alpha\left\|\mathbf{v}^{*}\right\|}+\frac{\alpha\left\langle\mathbf{c}-\mathbf{v}^{*}, \mathbf{v}^{*}\right\rangle}{\alpha\left\|\mathbf{v}^{*}\right\|} \\
& =\left\|\mathbf{v}^{*}\right\|+\frac{\left\langle\mathbf{c}-\mathbf{v}^{*}, \mathbf{v}^{*}\right\rangle}{\left\|\mathbf{v}^{*}\right\|} \\
& >\left\|\mathbf{v}^{*}\right\| .
\end{aligned}
$$

As such, for any $\mathbf{v}_{k}^{\alpha}=\alpha \mathbf{v}^{*}, \alpha>0$ we must have that

$$
\Delta_{k}\left(\mathbf{v}_{k}^{\alpha}\right)=\left\|\mathbf{v}^{*}\right\|
$$

Suppose $\mathbf{v}_{k}$ does not lie along $\mathbf{p}=\mathbf{v}^{*}$, i.e., $\mathbf{v}_{k} \neq \alpha \mathbf{v}^{*}, \alpha>0$. Let $\mathbf{v}_{k}=\mathbf{v}_{\|}+\mathbf{v}_{\perp}$ where $\mathbf{v}_{\|}$and $\mathbf{v}_{\perp}$ are parallel and perpendicular to $\mathbf{v}^{*}$ respectively. Consider $\mathcal{M}=C H\left(\mathcal{C}_{1}\right) \cap L$. Since the intersection of a hyperplane with a convex hull is a convex set, $\mathcal{M}$ is convex. Let $P(\mathbf{c}) \in \mathcal{M}$ be a constellation point lying on $L$. We can break $\mathbf{c}$ into components perpendicular and parallel to $\mathbf{v}^{*}$ as $\mathbf{c}=\mathbf{c}_{\|}+\mathbf{c}_{\perp}$. Since $\mathbf{c} \in L, \mathbf{c}_{\|}=\mathbf{v}^{*}$.

Now, by construction, the projection of the origin $O$ is the point $P$, which is an interior point of $\mathcal{M}$. If $P$ was exterior to $\mathcal{M}$, then $P$ will not be the closest point on $C H\left(\mathcal{C}_{1}\right)$ to $O$. If $P$ were not strictly interior to $\mathcal{M}$, but lay on the boundary of $\mathcal{M}$, then $P$ would be a constellation point in $\mathcal{C}_{1}$, which was treated in Case 1 . Note that evaluating $\operatorname{sgn}\left(\left\langle\mathbf{c}_{\perp}, \mathbf{v}_{\perp}\right\rangle\right)$ is geometrically equivalent to passing an $(N-1)$ dimensional hyperplane $L_{2}$ through the point $P$ orthogonal to $\mathbf{v}_{\perp}$ and determining to which side of $L_{2}$ the point $P(\mathbf{c})$ lies. Since $\mathcal{M}$ is a convex set, any hyperplane $L_{2}$ passing through an internal point will 
bi-partition it into two convex subsets. Therefore, there will always exist at least one point $\mathbf{c}^{*} \in \mathcal{M}$ such that $\left\langle\mathbf{c}_{\perp}^{*}, \mathbf{v}_{\perp}\right\rangle<0$. Hence, we must have

$$
\begin{aligned}
\left\langle\mathbf{c}^{*}, \mathbf{v}_{k}\right\rangle & =\left\langle\mathbf{c}_{\|}^{*}+\mathbf{c}_{\perp}^{*}, \mathbf{v}_{\|}+\mathbf{v}_{\perp}\right\rangle \\
& =\left\langle\mathbf{c}_{\|}^{*}, \mathbf{v}_{\|}\right\rangle+\left\langle\mathbf{c}_{\perp}^{*}, \mathbf{v}_{\perp}\right\rangle \\
& <\left\langle\mathbf{c}_{\|}^{*}, \mathbf{v}_{\|}\right\rangle \\
& =\left\|\mathbf{v}^{*}\right\|\left\|\mathbf{v}_{\|}\right\|
\end{aligned}
$$

and that

$$
\begin{aligned}
\frac{\left\langle\mathbf{c}^{*}, \mathbf{v}_{k}\right\rangle}{\left\|\mathbf{v}_{k}\right\|} & <\frac{\left\|\mathbf{v}^{*}\right\|\left\|\mathbf{v}_{\|}\right\|}{\left\|\mathbf{v}_{k}\right\|} \\
& <\left\|\mathbf{v}^{*}\right\|
\end{aligned}
$$

We then obtain that for any $\mathbf{v}_{k} \neq \alpha \mathbf{v}^{*}, \alpha>0$,

$$
\Delta_{k}\left(\mathbf{v}_{k}\right)<\left\|\mathbf{v}^{*}\right\| .
$$

From (28) and (31) it follows that $\Delta_{k, \text { opt }}=\left\|\mathbf{v}^{*}\right\|$. Combining cases 1 and 2, the result follows $\square$.

From Theorem 4.3, we observe that the maximum asymptotic efficiency of the detector for the $k^{\text {th }}$ user, $\eta_{k, \max }$ will be zero if the minimum distance from the origin to the convex hulls of the sub-constellations $\mathcal{C}\left(\mathbf{s}_{k}^{ \pm}\right)$is zero, i.e, if $C H\left(\mathcal{C}\left(\mathbf{s}_{k}^{ \pm}\right)\right)$overlap. We will show that this will happen if and only if $P\left(\mathbf{s}_{k}\right)$ belongs to the convex hull of the sub-constellation $\mathcal{C}\left(\mathbf{s}_{k}\right)=\sum_{i \neq k, b_{i}= \pm 1} b_{i} \mathbf{s}_{i}$.

Lemma 4.1: The convex hulls of sub-constellations $\mathcal{C}\left(\mathbf{s}_{k}^{+}\right)$and $\mathcal{C}\left(\mathbf{s}_{k}^{-}\right)$will overlap if and only if $P\left(\mathbf{s}_{k}\right) \in$ $C H\left(\mathcal{C}\left(\mathbf{s}_{k}\right)\right)$.

Proof: Since the signaling scheme used is BPSK, i.e., it is symmetric about the origin $O=P(\mathbf{0})$, the constellation points corresponding to any bit vector and its bitwise inverse will be radially symmetric about $O$. Thus the origin will be the midpoint of the line segment joining them. Therefore, the convex hulls of the signal constellations will also exhibit radial symmetry about the origin. Consider the $K$-user system with ordering $\mathcal{O}=\left\{\mathbf{s}_{1}, \mathbf{s}_{2}, \ldots, \mathbf{s}_{K}\right\}$, e.g., as shown in Figure 6 for $K=3$. Without loss of generality we will prove the stated result for the user signal $s_{1}$. Though the proof uses Figure 6 as an illustrative example, no assumption has been made that the number of users is three, or that the signal space is two-dimensional. The convex hull of sub-constellation $\mathcal{C}\left(\mathbf{s}_{1}\right)=\sum_{i=2, b_{i}= \pm 1}^{K} b_{i} \mathbf{s}_{i}$ is shown by solid lines and the convex hulls of the sub-constellations $\mathcal{C}\left(\mathbf{s}_{1}^{ \pm}\right)= \pm \mathbf{s}_{1}+\sum_{i=2, b_{i}= \pm 1}^{K} b_{i} \mathbf{s}_{i}$ are represented by dashed lines. Wherever applicable in the sequel $\mathbf{v}+P(\mathbf{s})$ denotes translation of the point $P(\mathbf{s})$ along the direction v. Note that, by construction, $\mathcal{C}\left(\mathbf{s}_{1}^{ \pm}\right)= \pm \mathbf{s}_{1}+\mathcal{C}\left(\mathbf{s}_{1}\right)$ and hence, $C H\left(\mathcal{C}\left(\mathbf{s}_{1}^{ \pm}\right)\right)= \pm \mathbf{s}_{1}+C H\left(\mathcal{C}\left(\mathbf{s}_{1}\right)\right)$, where + denotes translation along the direction of the vector $\pm \mathbf{s}_{1}$.

Proof of Lemma 4.1 (i): If $P\left(\mathbf{s}_{1}\right) \in C H\left(\mathcal{C}\left(\mathbf{s}_{1}\right)\right)$, then $\mathcal{C}\left(\mathbf{s}_{1}^{ \pm}\right)$overlap. 


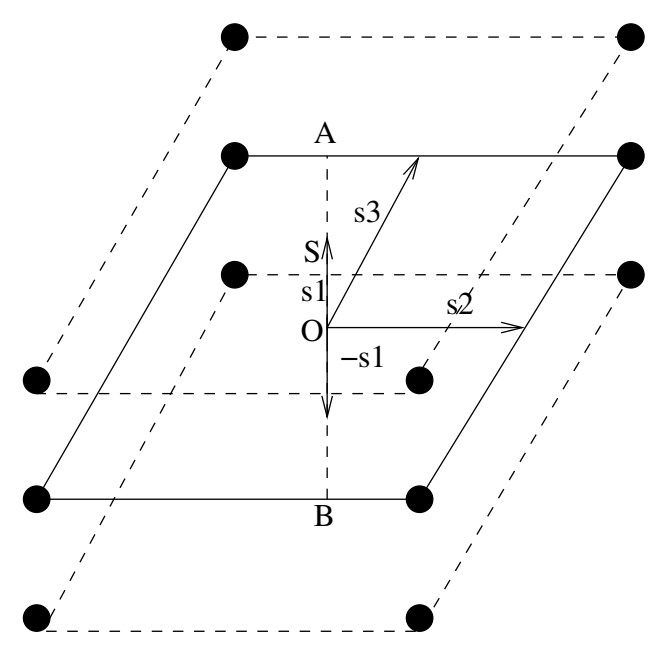

Fig. 6. Three-user system with ordering $\mathcal{O}=\left\{\mathbf{s}_{1}, \mathbf{s}_{2}, \mathbf{s}_{3}\right\}$.

Let $P\left(\mathbf{s}_{1}\right)=S$ and we extend the line $O S$ along the directions $\mathbf{s}_{1}$ and $-\mathbf{s}_{1}$ to intersect $C H\left(\mathcal{C}\left(\mathbf{s}_{1}\right)\right)$ at $A$ and $B$ respectively. Because of radial symmetry around the origin $O, O A=O B$. By construction $\left\|\mathbf{s}_{1}\right\|<O A$, which implies $\left\|-\mathbf{s}_{1}\right\|=\left\|\mathbf{s}_{1}\right\|<O A=O B$. Thus $P\left(-\mathbf{s}_{1}\right) \in C H\left(\mathcal{C}\left(\mathbf{s}_{1}\right)\right)$, which implies, $O=\mathbf{s}_{1}+P\left(-\mathbf{s}_{1}\right) \in \mathbf{s}_{1}+C H\left(\mathcal{C}\left(\mathbf{s}_{1}\right)\right)=C H\left(\mathcal{C}\left(\mathbf{s}_{1}^{+}\right)\right)$. Similarly, $0 \in C H\left(\mathcal{C}\left(\mathbf{s}_{1}^{-}\right)\right)$. Since $C H\left(\mathcal{C}\left(\mathbf{s}_{1}^{+}\right)\right)$ and $C H\left(\mathcal{C}\left(\mathbf{s}_{1}^{-}\right)\right)$both contain $O$, they must overlap. Therefore, if $\mathbf{s}_{1} \in C H\left(\mathcal{C}\left(\mathbf{s}_{1}\right)\right)$ then $C H\left(\mathcal{C}\left(\mathbf{s}_{1}^{+}\right)\right)$and $C H\left(\mathcal{C}\left(\mathbf{s}_{1}^{-}\right)\right)$overlap.

Proof of Lemma 4.1 (ii): $C H\left(\mathcal{C}\left(\mathbf{s}_{1}^{ \pm}\right)\right)$overlap only if $P\left(\mathbf{s}_{1}\right) \in C H\left(\mathcal{C}\left(\mathbf{s}_{1}\right)\right)$.

Suppose $C H\left(\mathcal{C}\left(\mathbf{s}_{1}^{+}\right)\right)$and $C H\left(\mathcal{C}\left(\mathbf{s}_{1}^{-}\right)\right)$overlap. Then there must exist at least one constellation point $M=P(\mathbf{p}), \mathbf{p}=-\mathbf{s}_{1}+\mathbf{s}$, where $\mathbf{s} \in \mathcal{C}\left(\mathbf{s}_{1}\right)$ such that $M \in C H\left(\mathcal{C}\left(\mathbf{s}_{1}^{+}\right)\right)$. By construction, the constellation point $M^{\prime}=P\left(\mathbf{p}_{c}\right)$ corresponding to the vector $\mathbf{p}_{c}=\left(\mathbf{s}_{1}-\mathbf{s}\right)$ also belongs to $C H\left(\mathcal{C}\left(\mathbf{s}_{1}^{+}\right)\right)$. From the definition of convex sets [50, p. 17], any point lying on the line segment between $M$ and $M^{\prime}$ belongs to $C H\left(\mathcal{C}\left(\mathbf{s}_{1}^{+}\right)\right)$. Therefore, from radial symmetry of linear constellations, $O$, the midpoint of $M$ and $M^{\prime}$ lies inside $C H\left(\mathcal{C}\left(\mathbf{s}_{1}^{+}\right)\right)$. Similarly, $O \in C H\left(\mathcal{C}\left(\mathbf{s}_{1}^{-}\right)\right) \Rightarrow-\mathbf{s}_{1}+P\left(\mathbf{s}_{1}\right) \in-\mathbf{s}_{1}+C H\left(\mathcal{C}\left(\mathbf{s}_{1}\right)\right)$, i.e., $P\left(\mathbf{s}_{1}\right) \in C H\left(\mathcal{C}\left(\mathbf{s}_{1}\right)\right)$.

Combining parts (i) and (ii), the result follows.

It is noteworthy that $\mathbf{s}_{k}$ must be a linear combination of $\left\{\mathbf{s}_{i}\right\}_{i=1, i \neq k}^{K}$, from the definition of convex sets [50, p. 17], for $P\left(\mathbf{s}_{k}\right)$ to lie in the convex hull of the sub-constellation generated by the other signals. Therefore, the condition $P\left(\mathbf{s}_{k}\right) \notin C H\left(\mathcal{C}\left(\mathbf{s}_{k}\right)\right)$, and hence $\eta_{k, \max }>0$, is trivially satisfied when the set of user signals are linearly independent. 


\section{MAXIMAL ASYMPTOTIC CONDITIONAL EFFICIENCY SUCCESSIVE INTERFERENCE CANCELLATION}

In this section, we will introduce the maximal asymptotic conditional efficiency (MACE) approach to successive detection. We derive the maximal asymptotic conditional efficiency successive interference canceller, henceforth referred to as the MACE-SIC detector that achieves the highest asymptotic conditional efficiency for a particular user among all possible SIC detectors for a given signal constellation and a given signal ordering. We define the asymptotic conditional multi-user efficiency for a given ordering of user signals as a quantitative measure of SIC detector performance based on the conditional probability of error at each stage of successive detection. Without loss of generality, let $\mathcal{O}=\left\{\mathbf{s}_{1}, \mathbf{s}_{2}, \ldots, \mathbf{s}_{K}\right\}$ be a given ordering of user signals for successive interference cancellation. Denote by $P_{i \mid \mathcal{O}}(\sigma)$ the conditional probability that the $i^{\text {th }}$ user's bit is correctly detected at the $i^{\text {th }}$ stage of successive detection, given that users with signals $\left\{\mathbf{s}_{1}, \mathbf{s}_{2}, \ldots, \mathbf{s}_{i-1}\right\}$ have been correctly detected in the first $(i-1)$ stages, i.e.,

$$
P_{i \mid \mathcal{O}}(\sigma)=P\left(\hat{b}_{i}=b_{i} \mid \hat{b}_{1}=b_{1}, \hat{b}_{2}=b_{2}, \ldots, \hat{b}_{i-1}=b_{i-1}\right) .
$$

Definition 5.1: Given an ordering $\mathcal{O}$, and a detector with conditional probability of error $P_{i \mid \mathcal{O}}(\sigma)$ for the $i^{\text {th }}$ user, the asymptotic conditional multi-user efficiency for the $i^{\text {th }}$ user, denoted as $\eta_{i \mid \mathcal{O}}$, is

$$
\eta_{i \mid \mathcal{O}}=\frac{1}{A_{i}^{2}} \lim _{\sigma \rightarrow 0} 2 \sigma^{2} \log \left(\frac{1}{P_{i \mid \mathcal{O}}(\sigma)}\right) .
$$

where $A_{i}^{2}=\left\|\mathbf{s}_{i}\right\|^{2}$ is the energy of the $i^{\text {th }}$ user. Accordingly, the asymptotic conditional effective energy is defined as the quantity $\eta_{i \mid \mathcal{O}} A_{i}^{2}$.

Many SIC detectors output bit estimates as $\hat{b}_{i}=\operatorname{sgn}\left(\mathbf{v}_{i}^{T}\left(\mathbf{y}-\sum_{j=1}^{i-1} \hat{b}_{j} \mathbf{s}_{j}\right)\right)$, i.e., at the $i^{\text {th }}$ stage, a linear detector is used on the received vector after subtracting off the interference of the user signals detected in previous stages. Thus, the maximum asymptotic conditional efficiency at the $i^{\text {th }}$ stage of detection is simply the maximum asymptotic efficiency achieved by a linear detector for the $i^{\text {th }}$ user in the reduced system $\tilde{\mathcal{S}}_{i}=\left\{\mathbf{s}_{j}\right\}_{j=i}^{K}$. Given an ordering $\mathcal{O}$, the maximum asymptotic conditional efficiency achievable by a SIC detector at the $i^{\text {th }}$ stage of detection, denoted as $\eta_{i, \max \mid \mathcal{O}}$, will be given as:

$$
\eta_{i, \max \mid \mathcal{O}}=\sup _{\mathbf{v}_{i} \in \Re^{N}} \max ^{2}\left(0, \frac{\mathbf{s}_{i}^{T} \mathbf{v}_{i}-\sum_{j=i+1}^{K}\left|\mathbf{s}_{j}^{T} \mathbf{v}_{i}\right|}{\| \mathbf{s}_{i}|||| \mathbf{v}_{i}||}\right),
$$

where $\max ^{2}(0, f(x))=\max \left(0,(f(x))^{2}\right)$ wherever applicable in the sequel.

From Theorem 4.3 it follows that (33) represents a convex optimization problem that seeks the minimum distance from the shifted origin $\mathbf{O}(i)=\sum_{k=1}^{i-1} \hat{b}_{k} \mathbf{s}_{k}$ to the convex hull of the $2^{K-i}$-point sub-constellation $\mathcal{C}(i)=\mathcal{C}\left(\left\{\mathbf{s}_{k}^{\operatorname{sign}\left(\hat{b}_{k}\right)}\right\}_{k=1}^{i-1}, \mathbf{s}_{i}^{ \pm}\right)$. The computational complexity of solving this convex optimization problem will be exponential in $(K-i)[51]$, yielding $\eta_{i, \max \mid \mathcal{O}}$ and

$$
\left\|\mathbf{v}_{i, \mathrm{opt} \mid \mathcal{O}}^{*}\right\|^{2}=d^{2}(\mathbf{O}(i), \mathcal{C}(i)) .
$$




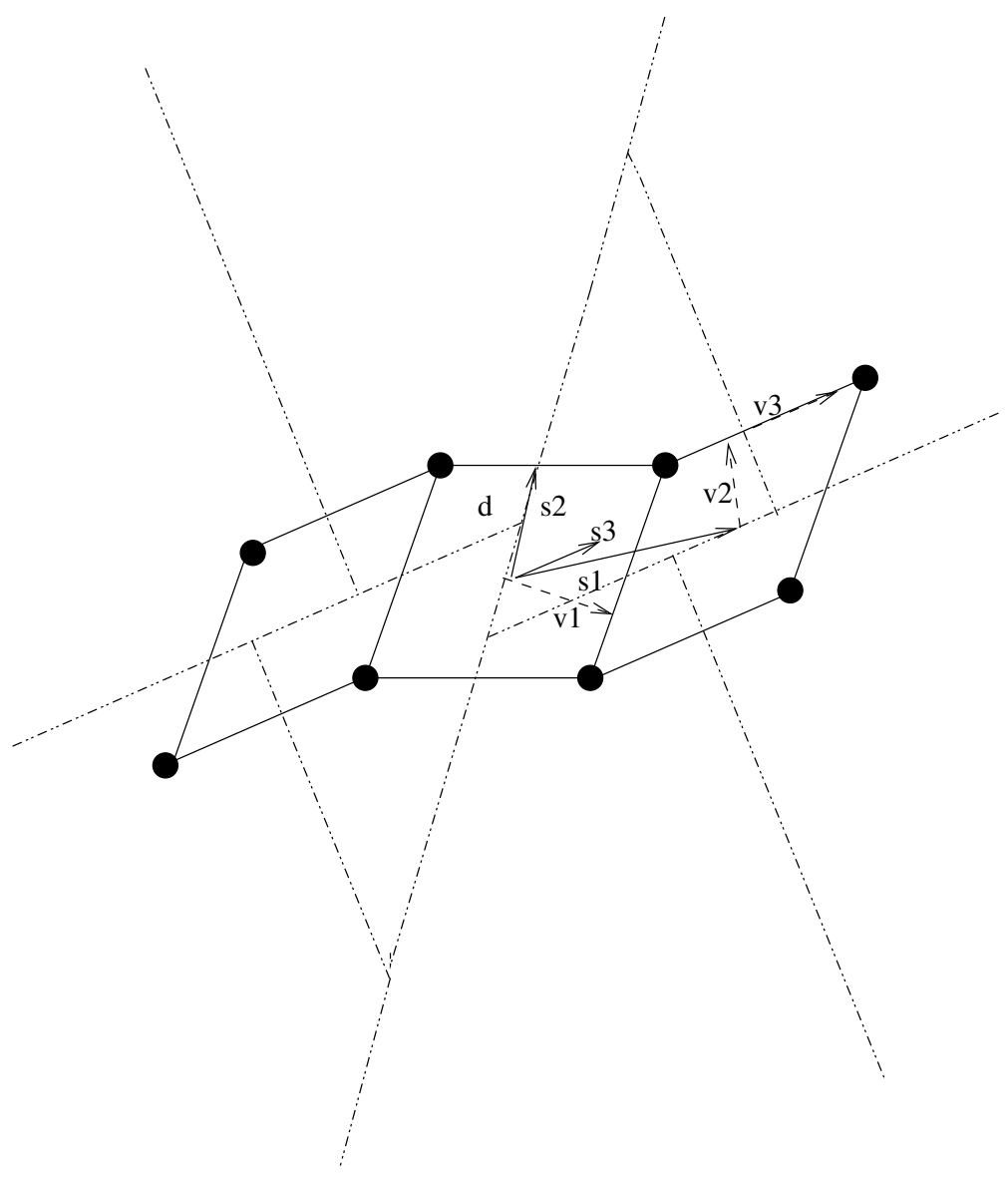

Fig. 7. Decision region of optimized SIC detector for the ordering $\left\{s_{1}, s_{2}, s_{3}\right\}$

Thus, the maximum asymptotic conditional efficiency successive interference canceller, given an ordering $\mathcal{O}$, henceforth referred to as the $\operatorname{MACE}-\operatorname{SIC}(\mathcal{O})$ detector, will output its bit estimates as

$$
\hat{b}_{i}=\operatorname{sgn}\left(\mathbf{v}_{i, \text { opt } \mid \mathcal{O}}^{T}\left(\mathbf{y}-\sum_{j=1}^{i-1} \hat{b}_{j} \mathbf{s}_{j}\right)\right) i=1,2, \ldots, K,
$$

where $\mathbf{v}_{i, \mathrm{opt} \mid \mathcal{O}}=\alpha \mathbf{v}_{i, \mathrm{opt} \mid \mathcal{O}}^{*}, \alpha>0$ is proportional to the minimum distance vector, $\mathbf{v}_{i, \mathrm{opt} \mid \mathcal{O}}^{*}$, from the shifted origin $\mathbf{O}(i)$ to the convex hull $\mathcal{C}(i)$.

The asymptotic conditional multi-user efficiency of the MACE-SIC $(\mathcal{O})$ detector at the $i^{\text {th }}$ stage, $\eta_{i, \max \mid \mathcal{O}}$, can therefore be expressed as

$$
\eta_{i, \max \mid \mathcal{O}}=\frac{d^{2}(\mathbf{O}(i), \mathcal{C}(i))}{\left\|\mathbf{s}_{i}\right\|^{2}}
$$

Figure 7 shows the complete decision regions for the MACE-SIC detector with the ordering $\left\{\mathbf{s}_{1}, \mathbf{s}_{2}, \mathbf{s}_{3}\right\}$. We now introduce an algorithm to generate the optimal ordering of user signals that maximizes the asymptotic conditional efficiency at each stage of successive detection. 


\section{A. Maximum asymptotic conditional efficiency ordering of user signals}

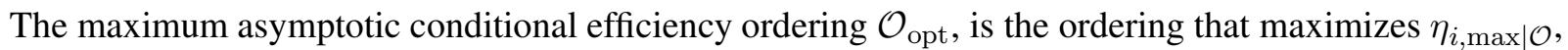
defined in Equation (33) among all possible orderings for each stage $i=1, \ldots, K$ in successive detection. Let $\eta_{\max }\left(\tilde{\mathcal{S}}, \mathbf{s}_{k}\right)$ denote the maximized asymptotic efficiency for a linear detector for the user with signal $\mathbf{s}_{k}$ in a multi-user system with $\tilde{\mathcal{S}}$ as the set of user signals. We can derive $\mathcal{O}_{\text {opt }}$ according to the following greedy algorithm:

1) Among $K$ possible choices for the user to be decoded first, choose the user signal $\mathbf{s}_{m_{1}}$ from the set $\tilde{\mathcal{S}}_{1}=\mathcal{S}$ such that

$$
\eta_{\max }\left(\tilde{\mathcal{S}}_{1}, \mathbf{s}_{m_{1}}\right)=\max _{k=1,2, \ldots, K} \eta_{\max }\left(\tilde{\mathcal{S}}_{1}, \mathbf{s}_{k}\right)
$$

2) Choose the second user signal $\mathbf{s}_{m_{2}}$ among the $(K-1)$ choices for the reduced system $\tilde{\mathcal{S}}_{2}=\tilde{\mathcal{S}}_{1}-\mathbf{s}_{m_{1}}$ such that

$$
\eta_{\max }\left(\tilde{\mathcal{S}}_{2}, \mathbf{s}_{m_{2}}\right)=\max _{k=1,2, \ldots, K, k \neq m_{1}} \eta_{k, \max }\left(\tilde{\mathcal{S}}_{2}, \mathbf{s}_{m_{2}}\right)
$$

3) Continue in this fashion such that at the $i^{\text {th }}$ stage we choose the user signal $\mathbf{s}_{m_{i}}$ among the $(K-i)$ choices left in $\tilde{\mathcal{S}}_{i}=\tilde{\mathcal{S}}_{i-1}-\mathbf{s}_{m_{i-1}}$ such that

$$
\eta_{\max }\left(\tilde{\mathcal{S}}_{i}, \mathbf{s}_{m_{i}}\right)=\max _{k=1,2, \ldots, K,\left\{k \neq m_{l}\right\}_{l=1}^{i-1}} \tilde{\eta}_{\max }\left(\tilde{\mathcal{S}}_{i}, \mathbf{s}_{k}\right)
$$

4) At the $K^{\text {th }}$ stage, the algorithm terminates yielding

$$
\mathcal{O}_{\mathrm{opt}}=\left\{\mathbf{s}_{m_{1}}, \mathbf{s}_{m_{2}}, \ldots, \mathbf{s}_{m_{K}}\right\}
$$

Note that $\mathcal{O}_{\text {opt }}$ maximizes the asymptotic conditional efficiency at each stage of successive detection, given the ordering this far, and does not necessarily maximize the overall asymptotic efficiency, or the asymptotic conditional efficiency for each (or any particular) user. However, it follows from (36) and (7) that the asymptotic efficiency $\eta_{k}$ for the $k^{\text {th }}$ user in the ordered set $\mathcal{O}$ will be lower bounded by the minimum asymptotic conditional effective energy, $\eta_{i} \mid(1,2, \ldots, i-1) A_{i}^{2}$, among all users preceding it, normalized by $A_{k}^{2}$. Therefore,

$$
\eta_{k} \geq \frac{1}{A_{k}^{2}} \min _{i}\left\{\eta_{i} \mid(1,2, \ldots, i-1) A_{i}^{2}\right\}_{i=1}^{k} .
$$

The ordering $\mathcal{O}_{\text {opt }}$ maximizes this lower bound by construction.

Complexity: It is noteworthy that the computational complexity of deriving $\mathcal{O}_{\text {opt }}$ is exponential in $K$, the number of users. A motivation behind this work is to trade off the complexity of setting up the SIC detector with the performance gain achieved by optimizing the asymptotic conditional efficiency at each stage of detection. The run-time complexity of the $\operatorname{MACE}-\operatorname{SIC}(\mathcal{O})$ detector is always linear in the number of users, i.e., $\mathcal{O}(K)$. As such, the MACE-SIC $(\mathcal{O})$ detector is suitable for stable communication systems with power control and a stable system of users, e.g., in satellite communications. In more dynamic multi-user systems, e.g., mobile cellular networks, the high complexity of setting up the detector when the system changes may render it impractical without modification. 
TABLE III

\section{The MACE-JSIC kernel at the $i^{\text {th }}$ stage}

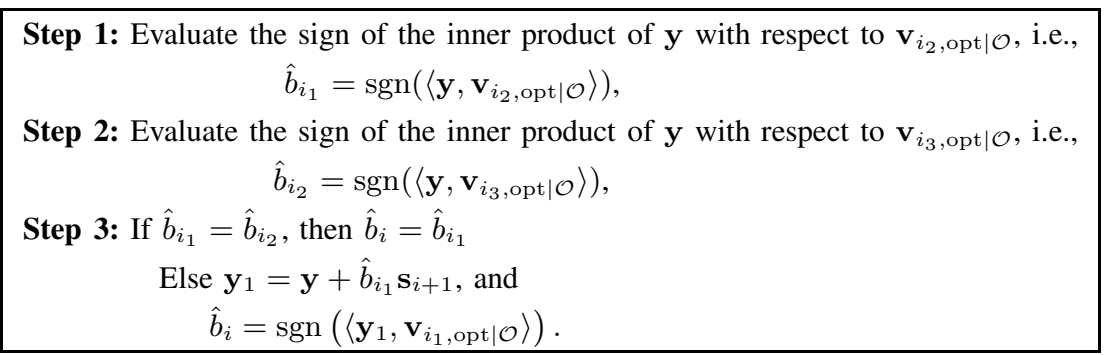

\section{MAXIMAL ASYMPTOTIC CONDITIONAL EFFICIENCY JOINT SUCCESSIVE INTERFERENCE CANCELLATION (JSIC) DETECTION}

In this section, we extend the MACE approach to JSIC detection and derive the JSIC detector that achieves the maximal asymptotic conditional efficiency at each stage of successive interference cancellation. Consider an ordering $\mathcal{O}=\left\{\mathbf{s}_{1}, \mathbf{s}_{2}, \ldots, \mathbf{s}_{K}\right\}$ of user signals. We set up the maximum asymptotic conditional efficiency JSIC detector, henceforth referred to as the MACE-JSIC $(\mathcal{O})$ detector, for a given ordering $\mathcal{O}$ as follows. We use $\eta_{\max }\left(\tilde{\mathcal{S}}, \mathbf{s}_{k}\right)$ to denote the maximized asymptotic efficiency for a linear detector for the user with signal $\mathbf{s}_{k}$ in the reduced system $\tilde{\mathcal{S}}$. Consider the four sub-constellations $\mathcal{C}_{\mathcal{O}}\left(\mathbf{s}_{i}^{ \pm}, \mathbf{s}_{i+1}^{ \pm}\right)$, where, e.g., $\mathcal{C}_{\mathcal{O}}\left(\mathbf{s}_{i}^{+}, \mathbf{s}_{i+1}^{+}\right)=\left\{\mathbf{s}_{i}+\mathbf{s}_{i+1}+\sum_{j=i+2}^{K} b_{j} \mathbf{s}_{j}: b_{j}= \pm 1\right\}$, at the $i^{\text {th }}$ stage of successive detection. Let $\mathbf{p}_{i_{1}}=\mathbf{s}_{i}+\mathbf{s}_{i+1}$ and $\mathbf{p}_{i_{2}}=\mathbf{s}_{i}-\mathbf{s}_{i+1}$. Let $\mathbf{v}_{i_{1}, \mathrm{opt} \mid \mathcal{O}}, \mathbf{v}_{i_{2} \text {,opt } \mid \mathcal{O}}$ and $\mathbf{v}_{i_{3}, \mathrm{opt} \mid \mathcal{O}}$ be the optimized vectors for linear detection that achieve $\eta_{\max }\left(\tilde{\mathcal{S}}_{i_{1}}, \mathbf{s}_{i}\right), \eta_{\max }\left(\tilde{\mathcal{S}}_{i_{1}}, \mathbf{p}_{i_{1}}\right)$ and $\eta_{\max }\left(\tilde{\mathcal{S}}_{i_{1}}, \mathbf{p}_{i_{2}}\right)$ respectively, in the reduced systems $\tilde{\mathcal{S}}_{i_{1}}=\left\{\mathbf{s}_{i}, \mathbf{s}_{i+2}, \ldots, \mathbf{s}_{K}\right\}, \tilde{\mathcal{S}}_{i_{2}}=\left\{\mathbf{p}_{i_{1}}, \mathbf{s}_{i+2}, \ldots, \mathbf{s}_{K}\right\}$ and $\tilde{\mathcal{S}}_{i_{3}}=$ $\left\{\mathbf{p}_{i_{2}}, \mathbf{s}_{i+2}, \ldots, \mathbf{s}_{K}\right\}$ respectively. The MACE-JSIC $(\mathcal{O})$ detector uses the vectors $\mathbf{v}_{i_{1}, \text { opt } \mid \mathcal{O}}, \mathbf{v}_{i_{2}, \text { opt } \mid \mathcal{O}}$ and $\mathbf{v}_{i_{3}, \mathrm{opt} \mid \mathcal{O}}$ instead of $\mathbf{s}_{i}, \mathbf{p}_{i_{1}}$ and $\mathbf{p}_{i_{2}}$ respectively at the $i^{\text {th }}$ stage of interference cancellation. Since we are using sub-constellations, and not constellation points in the two-user MACE-JSIC kernel, we need to consider both $\mathbf{p}_{i_{1}}$ and $\mathbf{p}_{i_{2}}$, rather than $\mathbf{p}_{i}$ in the JSIC kernel and therefore, have three, instead of two inner product operations in the MACE-JSIC kernel as shown in Table III.

From Theorem 4.2 it can be shown that the asymptotic conditional efficiency $\eta_{i, \max \mid \mathcal{O}}$ of the MACE$\operatorname{JSIC}(\mathcal{O})$ detector, will be given by

$$
\eta_{i, \max \mid \mathcal{O}}=\min \left\{\eta_{\max }\left(\tilde{\mathcal{S}}_{i_{1}}, \mathbf{s}_{i}\right), \eta_{\max }\left(\tilde{\mathcal{S}}_{i_{2}}, \mathbf{p}_{i_{1}}\right), \eta_{\max }\left(\tilde{\mathcal{S}}_{i_{3}}, \mathbf{p}_{i_{2}}\right)\right\} .
$$

The ordering $\mathcal{O}_{\text {opt }}$ that achieves the highest asymptotic conditional efficiency at each stage among all possible orderings can then be achieved through a similar algorithm to that given in Section V-A. At each stage $i$, we choose the two user signals $\mathbf{s}_{m_{i_{1}}}$ and $\mathbf{s}_{m_{i_{2}}}$ over the $C_{2}^{K-i+1}$ possible choices such that 
the quantity

$$
\tilde{\eta}_{i}=\min \left\{\eta_{\max }\left(\tilde{\mathcal{S}}_{l_{i_{1}}}, \mathbf{s}_{l_{i_{1}}}\right), \eta_{\max }\left(\tilde{\mathcal{S}}_{l_{i_{2}}}, \mathbf{p}_{l_{i_{1}}}\right), \eta_{\max }\left(\tilde{\mathcal{S}}_{l_{i_{3}}}, \mathbf{p}_{l_{i_{2}}}\right)\right\} .
$$

is maximized $\forall \mathbf{s}_{l_{i_{1}}}, \mathbf{s}_{l_{i_{2}}} \in \tilde{\mathcal{S}}_{i}=\mathcal{S}-\left\{\mathbf{s}_{m_{j}}\right\}_{j=1}^{i-1}$, where $\tilde{\mathcal{S}}_{l_{i_{1}}}=\tilde{\mathcal{S}}_{i}-\mathbf{s}_{l_{i_{2}}}, \tilde{\mathcal{S}}_{l_{i_{2}}}=\tilde{\mathcal{S}}_{l_{i_{1}}}-\mathbf{s}_{l_{i_{1}}}+\mathbf{p}_{l_{i_{1}}}$, and $\tilde{\mathcal{S}}_{l_{3}}=\tilde{\mathcal{S}}_{l_{i_{1}}}-\mathbf{s}_{l_{i_{1}}}+\mathbf{p}_{l_{i_{2}}}$. Figure 8 shows the decision regions for a MACE-JSIC detector for a given ordering in a four-user system in two dimensions. Though the run-time complexity of MACE-JSIC $(\mathcal{O})$ and MACE-SIC $(\mathcal{O})$ detector are linear in the number of users, the MACE-JSIC $(\mathcal{O})$ detector performs three inner product operations at each stage compared to the MACE-SIC $(\mathcal{O})$ detector, which performs only one inner product operation. The design complexity of the MACE-JSIC $(\mathcal{O})$ detector is exponential in $K$, the number of users, and its run-time complexity, though linear in the number of users, is greater than the run-time complexity of the $\operatorname{MACE}-\operatorname{SIC}(\mathcal{O})$ detector. We now show that this increase in run-time complexity is compensated by an increase in the asymptotic conditional multi-user efficiency at each stage of successive interference cancellation.

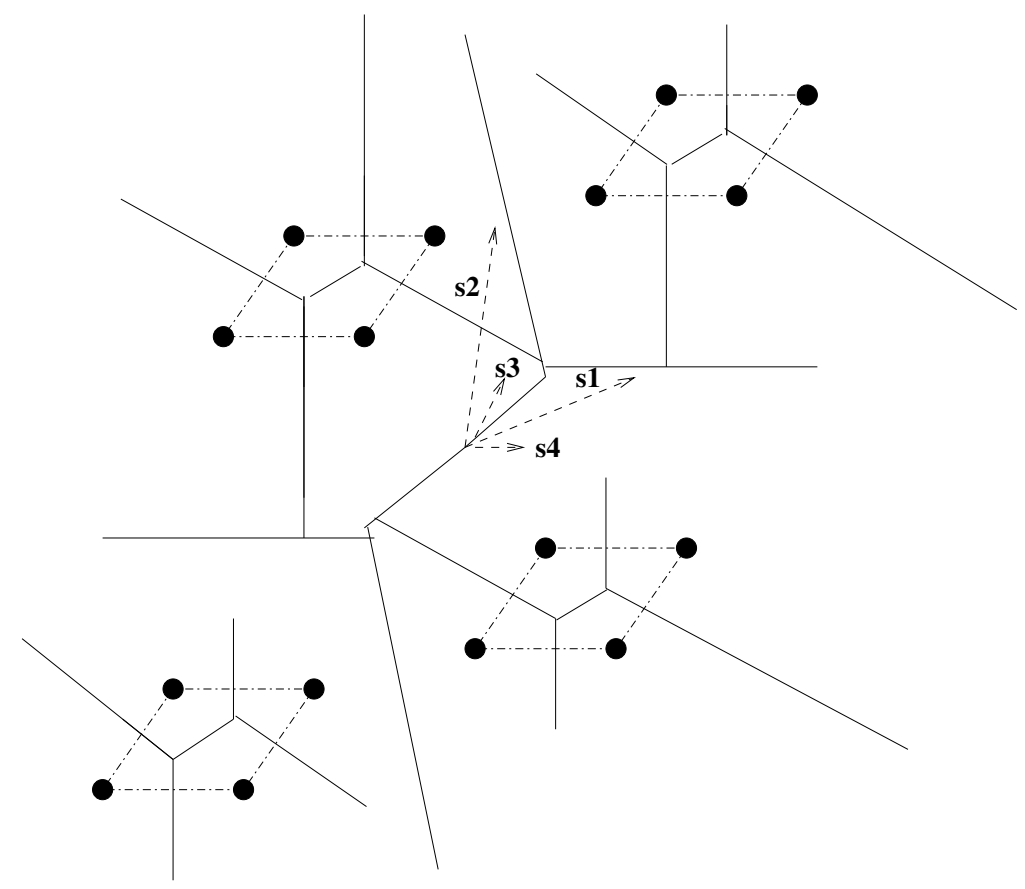

Fig. 8. Decision regions of MACE-JSIC $(\mathcal{O})$ detector with ordering $\left\{\mathbf{s}_{1}, \mathbf{s}_{2}, \mathbf{s}_{3}, \mathbf{s}_{4}\right\}$ for four-user system

Lemma 6.1: For a given convex hull ordering $\mathcal{O}$, the MACE-JSIC $(\mathcal{O})$ detector always achieves higher asymptotic conditional efficiency for every user than the corresponding $\operatorname{MACE}-\operatorname{SIC}(\mathcal{O})$ detector.

Proof: At the $i^{\text {th }}$ stage of interference cancellation, the asymptotic conditional efficiency of user $i$ given $\left\{b_{1}, b_{2}, \ldots, b_{i-1}\right\}$ is the minimum of $\left\|\mathbf{v}_{i_{1}, \mathrm{opt} \mid \mathcal{O}}\right\|^{2},\left\|\mathbf{v}_{i_{2}, \mathrm{opt} \mid \mathcal{O}}\right\|^{2}$ and $\left\|\mathbf{v}_{i_{3}, \mathrm{opt} \mid \mathcal{O}}\right\|^{2}$, normalized by $A_{i}^{2}$, i.e.,

$$
\eta_{M A C E-J S I C}\left(i \mid b_{1}, b_{2}, \ldots, b_{i-1}\right)=\frac{1}{A_{i}^{2}} \min \left(\left\|\mathbf{v}_{i_{1}, \mathrm{opt} \mid \mathcal{O}}\right\|^{2},\left\|\mathbf{v}_{i_{2}, \mathrm{opt} \mid \mathcal{O}}\right\|^{2},\left\|\mathbf{v}_{i_{3}, \mathrm{opt} \mid \mathcal{O}}\right\|^{2}\right)
$$


Let $\mathbf{v}_{i, M A C E-S I C}$ denote the minimum distance vector between $C H\left(\mathcal{C}\left(\mathbf{s}_{i}^{+}\right)\right)$and $C H\left(\mathcal{C}\left(\mathbf{s}_{i}^{-}\right)\right)$. Then,

$$
\eta_{M A C E-S I C}\left(i \mid b_{1}, b_{2}, \ldots, b_{i-1}\right)=\frac{\left\|\mathbf{v}_{i, M A C E-S I C}\right\|}{A_{i}^{2}} .
$$

Since $C H\left(\mathcal{C}\left(\mathbf{s}_{i}^{ \pm}, \mathbf{s}_{i+1}^{ \pm}\right) \subset C H\left(\mathcal{C}\left(\mathbf{s}_{i}^{ \pm}\right)\right)\right.$, and the minimum distance between subsets of convex sets is always greater than or equal to the distance between the convex sets, we must have

$$
\mathbf{v}_{i, M A C E-S I C} \leq \min \left(\left\|\mathbf{v}_{i_{1}, \mathrm{opt} \mid \mathcal{O}}\right\|^{2},\left\|\mathbf{v}_{i_{2}, \mathrm{opt} \mid \mathcal{O}}\right\|^{2},\left\|\mathbf{v}_{i_{3}, \mathrm{opt} \mid \mathcal{O}}\right\|^{2}\right) .
$$

Combining (40), (41) and (42) the result follows $\square$.

\section{PERFORMANCE BOUNDS ON MULTI-LEVEL JOINT SUCCESSIVE INTERFERENCE CANCELLATION}

We have so far considered joint successive interference cancellation considering two users at a time from an ordered set of user signals $\mathcal{O}=\left\{\mathbf{s}_{1}, \mathbf{s}_{2}, \ldots, \mathbf{s}_{K}\right\}$. We can extend this concept and derive a family of maximal asymptotic conditional efficiency successive interference cancellers where, at each stage, we consider more than two users at a time. In the previous section, we have shown that the increase in runtime complexity by choosing MACE-JSIC over MACE-SIC is compensated by a corresponding increase in asymptotic conditional efficiency. In this section, we derive a design measure that quantifies this tradeoff between complexity and performance by providing a bound over the performance gain achieved by considering more user signals in the MACE-JSIC kernel.

At the $i^{\text {th }}$ stage of successive detection, we consider the $2^{m}$ sub-constellations, $m$ being a design parameter, denoted as $\mathcal{C}_{m}$ and given as, $\mathcal{C}_{m}=\mathcal{C}\left(\mathbf{s}_{i}^{ \pm}, \ldots, \mathbf{s}_{i+m-1}^{ \pm}\right)$. Let us denote the sub-constellations belonging to $\mathcal{C}_{m}$ that have the $i^{\text {th }}$ user's bit set to \pm 1 as $\mathcal{C}_{m}\left(\mathbf{s}_{i}^{ \pm}\right)$. The $i^{\text {th }}$ user's bit is decoded based on the joint information between users with signals $\left\{\mathbf{s}_{i}, \mathbf{s}_{i+1}, \ldots, \mathbf{s}_{i+m-1}\right\}$. The asymptotic conditional efficiency for the $i^{\text {th }}$ user for the $\operatorname{MACE}-\operatorname{JSIC}(m)$ detector, denoted as $\eta_{i}(m)$ will be given by the minimum of the minimum distance between the convex hulls of any two sub-constellations belonging to $\mathcal{C}_{m}\left(\mathbf{s}_{i}^{+}\right)$and $\mathcal{C}_{m}\left(\mathbf{s}_{i}^{-}\right)$respectively, normalized by the energy, $A_{i}^{2}=\left\|\mathbf{s}_{i}\right\|^{2}$ of the $i^{\text {th }}$ user. Since subconstellations belonging to $\mathcal{C}_{m}\left(\mathbf{s}_{i}^{+}\right)$are a subset of $\mathcal{C}_{m-1}\left(\mathbf{s}_{i}^{+}\right)$, i.e., as $m$ increases, we partition each sub-constellation into smaller and smaller sub-constellations. Since the minimum distance between the subsets of convex sets is always greater than the minimum distance between the convex sets, we must have,

$$
\eta_{i}(1) \leq \eta_{i}(2) \leq \ldots \eta_{i}(m)
$$

However, the run-time complexity of the MACE-JSIC $(m)$ detector will have $2^{2(m-1)}$ inner products, i.e., it increases exponentially with $m$. Also, the design complexity of the MACE-JSIC $(m)$ detector will be exponential in $K$ and $m$. Therefore, the gain in $\eta_{i}(m)$ as $m$ increases is offset by an exponential increase in complexity. This motivates us to derive bounds on $\eta_{i}(m)$ and determine a quantitative measure to compare the performance of the MACE-JSIC $(m)$ detectors as $m$ increases. Let us start with the basic 
MACE-JSIC(2) detector and compare its performance with respect to the MACE-SIC, i.e., the MACEJSIC(1) detector.

Let us denote $\alpha_{i}(m)=\sqrt{\eta_{i}(m) A_{i}^{2}}$, where $A_{i}^{2}=\left\|\mathbf{s}_{i}\right\|^{2}$ is the energy of the $i^{\text {th }}$ user. To compare between $\eta_{i}\left(m_{1}\right)$ and $\eta_{i}\left(m_{2}\right)$ it is sufficient to compare $\alpha_{i}\left(m_{1}\right)$ and $\alpha_{i}\left(m_{2}\right)$. Let $d(1)$ be the minimum distance from the origin to the convex hull of the sub-constellation $\mathcal{C}_{1}\left(\mathbf{s}_{i}^{+}\right)$. Therefore

$$
\alpha_{i}(1)=d(1)
$$

Now, $d(1)$ is the solution to the convex optimization

$$
d(1)=\min _{\lambda_{j} \geq 0, \sum_{j=1}^{2 K-1} \lambda_{j}=1}\left\|\sum_{j=1}^{2^{K-1}} \lambda_{j} \mathbf{c}_{j}\right\|, \mathbf{c}_{j} \in \mathcal{C}\left(\mathbf{s}_{i}^{+}\right), j=1, \ldots, 2^{K-1} .
$$

Let $\Delta(++,--)$ denote the minimum distance between the convex hulls of the sub-constellations $\mathcal{C}\left(\mathbf{s}_{i}^{+}, \mathbf{s}_{i+1}^{+}\right)$and $\mathcal{C}\left(\mathbf{s}_{i}^{-}, \mathbf{s}_{i+1}^{-}\right)$. Similarly, we define $\Delta(+-,-+), \Delta(+-,--)$ and $\Delta(++,-+)$ as the minimum distance between the corresponding sub-constellations. For linear systems, we must have $\Delta(+-,--)=\Delta(++,+-)$ from symmetry considerations. Therefore, we must have,

$$
\alpha_{i}(2)=\frac{1}{2} \min \{\Delta(++,--), \Delta(+-,-+), \Delta(++,--)\} .
$$

Let $d(2)=\frac{1}{2} \min (\Delta(++,--), \Delta(+-,-+))$. For linear systems, $d(2)$ is the minimum distance from the origin $O$ to the convex hull of the sub-constellation

$$
\mathcal{C}(1)=\arg \min \{\Delta(++,--), \Delta(+-,-+)\}
$$

that has $b_{i}=1$, i.e.,

$$
d(2)=\min _{\beta_{j} \geq 0, \sum_{j=1}^{2^{K-2}} \beta_{j}=1}\left\|\sum_{j=1}^{2^{K-2}} \beta_{j} \mathbf{c}_{j}\right\|, \mathbf{c}_{j} \in \mathcal{C}(1), j=1, \ldots, 2^{K-2} .
$$

Also, by construction, we must have $\alpha_{i}(2) \leq d(2)$. Now, let $\Lambda(1)=\left\{\lambda_{j}^{*}\right\}_{j=1}^{2^{K-1}}$ be the set $\left\{\lambda_{j}\right\}_{j=1}^{2^{K-1}}$ that provides the solution to Equations (45). Let us denote $\Delta_{\Lambda(1)}(2)=\left\|\sum_{j=1}^{2^{K-2}} \lambda_{j}^{*} \mathbf{c}_{j}\right\|, \mathbf{c}_{j} \in \mathcal{C}(1), j=$ $1, \ldots, 2^{K-2}$. Note that without loss of generality, we have assumed $\left\{\mathbf{c}_{j}\right\}_{j=1}^{2^{K-2}} \in \mathcal{C}(1)$. Therefore, we have that

$$
\begin{aligned}
\Delta_{\Lambda(1)}(2) & =\left\|\sum_{j=1}^{2^{K-2}} \lambda_{j}^{*} \mathbf{c}_{j}\right\| \\
& =\left\|\sum_{j=1}^{2^{K-2}} \frac{\lambda_{j}^{*}}{\sum_{j=1}^{2^{K-2}} \lambda_{j}^{*}}\left(\sum_{j=1}^{2^{K-2}} \lambda_{j}^{*}\right) \mathbf{c}_{j}\right\| \\
& =\frac{1}{\gamma(2)}\left\|\sum_{j=1}^{2^{K-2}}{\tilde{\lambda^{*}}}_{j} \mathbf{c}_{j}\right\|,
\end{aligned}
$$


where $\gamma(2)=\frac{1}{\sum_{j=1}^{2 K-2} \lambda_{j}^{*}}, \tilde{\lambda}^{*}{ }_{j}=\gamma(2) \lambda_{j}^{*}$, and $\sum_{j=1}^{2^{K-2}} \tilde{\lambda}^{*}{ }_{j}=1$. Therefore, we obtain,

$$
\begin{aligned}
\Delta_{\Lambda(1)}(2) \gamma(2) & =\left\|\sum_{j=1}^{2^{K-2}} \tilde{\lambda}^{*} \mathbf{c}_{j}\right\|, \\
& \geq d(2) .
\end{aligned}
$$

Combining the above inequality with (44) and (43) we have the following inequality:

$$
d(1) \leq d(2) \leq \Delta_{\Lambda(1)}(2) \gamma(2)
$$

Similarly, we can set up the sequence $\left\{\Delta_{\Lambda(1)}(2), \Delta_{\Lambda(1)}(3), \ldots, \Delta_{\Lambda(1)}(m)\right\}$ and arrive at the relation:

$$
d(1) \leq d(2) \leq \ldots d(m) \leq \Delta_{\Lambda(1)}(m) \gamma(m)
$$

which leads to the relationship

$$
\alpha_{i}(1) \leq \alpha_{i}(2) \leq \ldots \alpha_{i}(m) \leq \Delta_{\Lambda(1)}(m) \gamma(m) .
$$

Thus the ratio $r(m)=\frac{\Delta_{\Lambda(1)}(m) \gamma(m)}{d(1)}$ can be used as a quantitative measure of the performance gain and as a metric to determine whether or not it is worth the increase in design or runtime complexity by further increasing $m$.

VIII. Simulation RESUltS

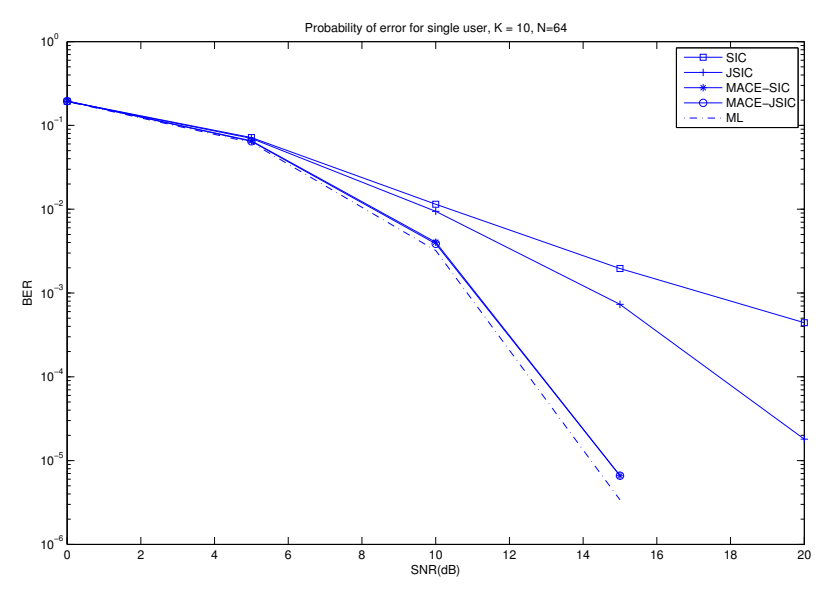

Fig. 9. Single-user BER in a representative ten-user system averaged over all users

In this section, we present numerical evidence supporting our theoretical assertions in previous sections. All user signals random Gaussian sequences of length $N$, and all bit streams were random BPSK sequences. The performance of the JSIC, MACE-SIC and MACE-JSIC detectors have been tested against 


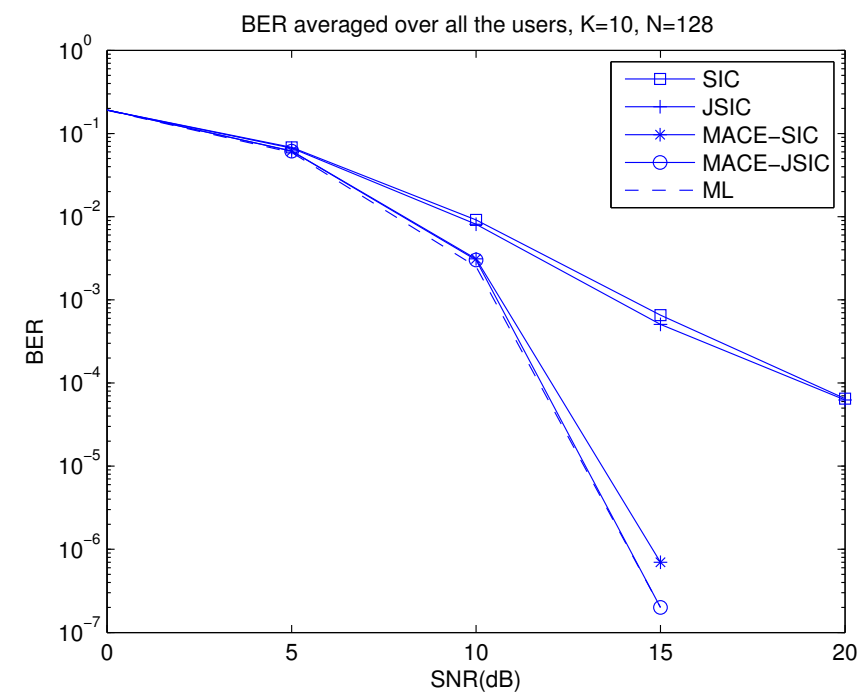

Fig. 10. Single-user BER in a representative ten-user system averaged over all users

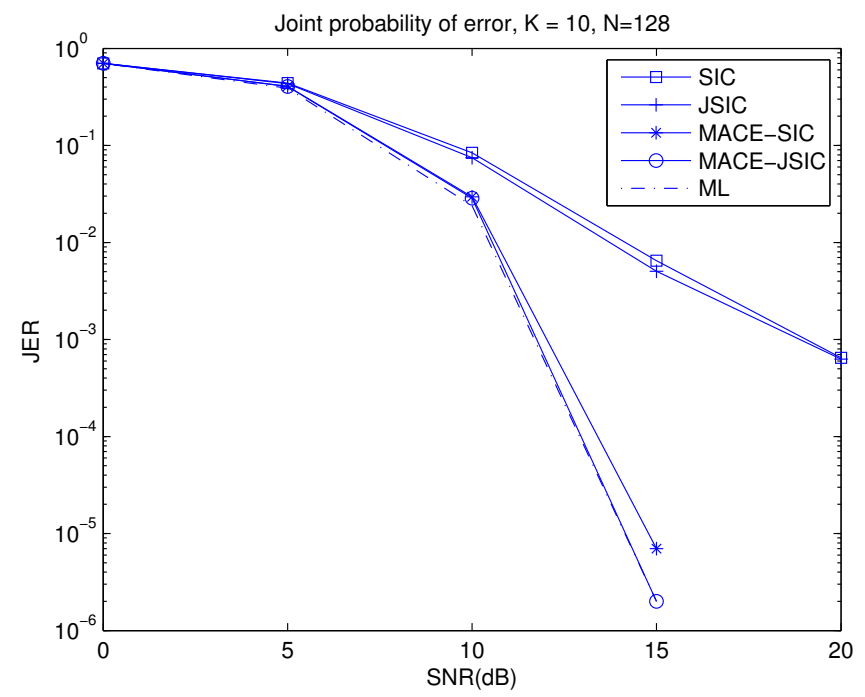

Fig. 11. Joint error rate (JER) in a representative ten-user system

the conventional SIC detector, and the performance of the ML detector has been provided as a lower bound. Numerical simulations were run on several multi-user systems and the bit-error-rate (BER) of each user was averaged over the user signals and bit streams. Figures 9 and 10 show the average BER (i.e., the bit error rate averaged over all users) for a random ten-user system with $N=64$ and $N=128$ respectively. Figure 11 shows the joint error rate (JER) respectively in a random ten-user system with $N=128$. We observe that the MACE-SIC and MACE-JSIC consistently outperform the conventional 


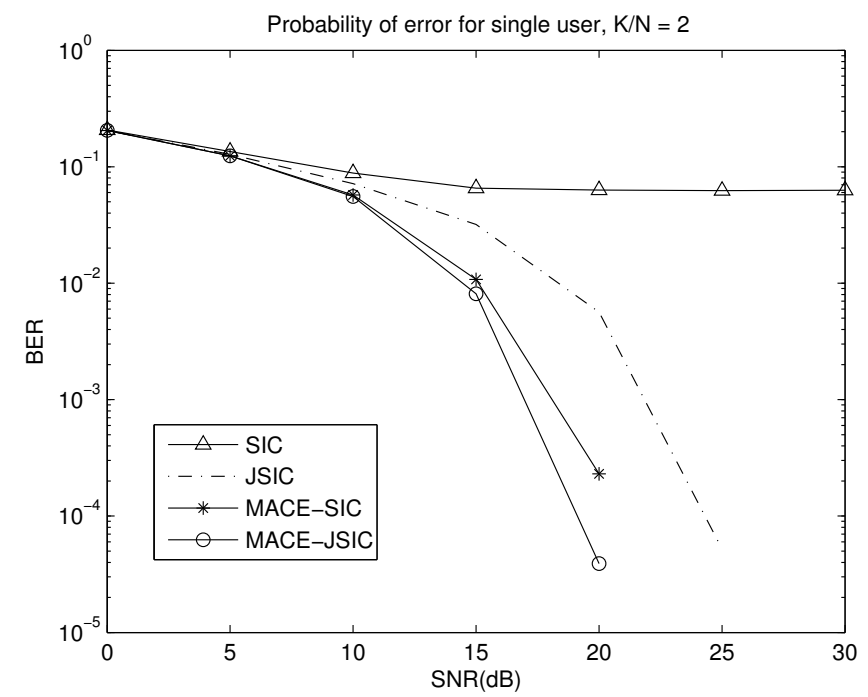

Fig. 12. Single-user BER in a representative four-user system

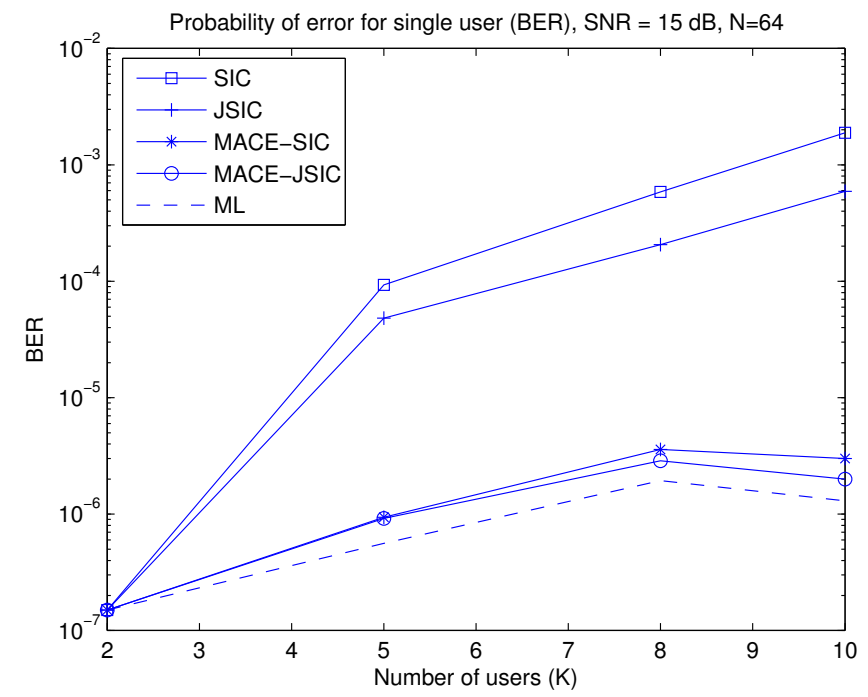

Fig. 13. BER comparison for SIC, JSIC, MACE-SIC, MACE-JSIC and ML detectors for SNR=15dB plotted against number of users

SIC and JSIC detectors and the performance of MACE-JSIC is generally superior to that of MACE-SIC, though at high SNR their performance is quite close. We also observe that on average the conventional SIC and JSIC detectors are not near-far resistant, but the MACE-SIC and MACE-JSIC detectors show robustness against multi-user interference since the conditional multi-user efficiency has been optimized at each stage. Figure 12 shows the single user performance for a particular over-loaded four-user system, 
i.e., systems where the number of users, $K$, is greater than the number of dimensions $N$, and therefore, the user signals $\mathcal{S}$ form a linearly dependent set. Figure 13 also shows simulations in which the SNR is held fixed at $15 \mathrm{~dB}$ and the BER of the proposed detectors are observed over an increasing number of users.

\section{CONCLUSION}

We have introduced joint successive interference cancellation as an improvement over conventional successive interference cancellation. We also presented an approach to successive interference cancellation that exploits the structure of the multi-user signal constellation to annul the effect of the closest interferer in an ordered set of users. Defining the conditional efficiency of a successive interference canceller, we determined the SIC and JSIC detectors with the highest asymptotic conditional efficiency for a given ordered set of users. We have also derived the maximum asymptotic conditional efficiency ordering of user signals that achieves the highest asymptotic conditional efficiency at each stage of successive interference cancellation. The performance of the MACE-JSIC detector is shown to be strictly higher than that of the MACE-SIC detector. Based on this principle, we also proposed a family of joint successive interference cancellers that achieve higher performance with an increase in complexity. By quantifying the trade-off between detector complexity and performance gain, in terms of the signal constellation structure, higher performance gains in conditional probability of error can be achieved at high SNR. The successive interference cancellation techniques introduced in this work assume perfect knowledge of the signal constellation, and are practical for implementation in stable multi-user systems with good power control, e.g., in satellite communications. However, in dynamic multi-user systems the signal constellation may be continuously changing due to mobility with abrupt changes as users enter or leave the system. The user signals also may not be perfectly synchronized or may change over time due to fading or imperfect power control. The high design complexity of MACE detection can also be significantly reduced for multi-user systems with reasonable restrictions on the signal constellation, e.g., when the user signals are linearly independent.

\section{REFERENCES}

[1] S. Verdú, Multiuser Detection. Cambridge University Press, 1998.

[2] R. Lupas and S. Verdú, "Linear multi-user detectors for synchronous code-division multiple-access channels," IEEE Trans. Info. th., vol. 35, no. 1, Jan. 1989.

[3] U. Madhow and M. Honig, "Mmse interference suppression for direct-sequence spread spectrum CDMA," IEEE Trans. on Commun., vol. 42, pp. 3178-3188, Dec. 1994.

[4] H. Poor and S. Verdú, "Probability of error in MMSE multiuser detection," IEEE Trans. Info. Th., vol. 43, pp. 858-871, May 1997.

[5] E. Dahlman and K. Jamal, "Multi-stage serial interference cancellation for DS-CDMA," in IEEE 46 ${ }^{\text {th }}$ Vehicular Technology Conference, Atlanta, GA, May 1996. 
[6] A. Johansson and A. Svensson, "Successive interference cancellation schemes in multi-rate ds/cdma systems," in Workshop records, $5^{\text {th }}$ WINLAB Workshop on $3^{\text {rd }}$ generation wireless information networks, vol. 3, 1995, pp. 155-174.

[7] — , "Multi-stage interference cancellation in multi-rate DS/CDMA systems," in Proc. $6^{\text {th }}$ IEEE International Symposium on Personal, Indoor and Mobile Radio Communications, vol. 3, Sept. 1995, pp. 965-969.

[8] A. Duel-Hallen, "Decorrelating decision feedback multiuser detector for synchronous code-division multiple-access channel," IEEE Trans.Commun., vol. 41, pp. 285-290, Feb. 1993.

[9] A. Duel-Hallen, J. Holtzman, and Z. Zvonar, "Multiuser detection for CDMA systems," IEEE Trans.Commun., vol. 43, no. 2/3/4, p. 4658, Apr. 1995.

[10] A. Duel-Hallen, "A family of decision feedback multiuser detectors for asynchronous code-division multiple-access channels," IEEE Trans.Commun., vol. 43, no. 2/3/4, pp. 421-434, 1995.

[11] D. Divsalar, M. Simon, and D. Raphaeli, "Improved parallel interference cancellation for CDMA," IEEE Trans. on Commun., vol. 46, no. 2, pp. 258-268, Feb. 1998.

[12] M. Varanasi and B. Aazhang, "Near-optimum detection in synchronous code-division multiple access systems," IEEE Trans. Commun., vol. 39, no. 5, May 1991.

[13] H. Sari, F. Vanhaverbeke, and M. Moeneclaey, "Combined TDMA/OCDMA with iterative multistage detection," in Proc. IEEE Intl. Conf. Communications, vol. 2, New Orleans, LA, June 2000.

[14] G. Barriac and U. Madhow, "PASIC: A new paradigm for low-complexity multiuser detection," in Proc. CISS 2001, Baltimore, MD, March 21-23 2001.

[15] S. Verdú, "Multiuser detection," in Advances in Statistical Signal Processing: Signal Detection, H. Poor and J. Thomas, Eds. JAI Press, 1993, pp. 369-410.

[16] S. Moshavi, "Multi-user detection for DS-CDMA communications," in IEEE Commun. Mag., May 1996, pp. 124-136.

[17] Z. Xie, R. Short, and C. Rushforth, "A family of suboptimum detectors for coherent multiuser communications," IEEE J. Select. Areas Commun., vol. 8, pp. 683-690, May 1990.

[18] E. Fain and M. Varanasi, "Cyclic decision feedback multiuser sequence detection," in Proc.32 ${ }^{\text {nd }}$ Annu. Allerton Conf. Communication, Control, and Computing, vol. 3, Oct. 1994, pp. 372-381.

[19] M. Varanasi, "Group detection for synchronous gaussian code-division multiple-access channels," IEEE Trans. Inform. Theory, vol. 41, pp. 1083-1096, July 1995.

[20] Y. Sun, "Local maximum likelihood multiuser detection," in Proc. CISS 2001, Baltimore, MD, March 21-23 2001, pp. $7-12$.

[21] S. Buzzi, M. Lops, and G. Ricci, "A new group detection strategy for DS-CDMA systems," in Proc. IEEE Int. Symp. Information Theory, Sorrento, Italy, June 2000, p. 357.

[22] S. Buzzi, M. Lops, A. Pauciullo, and G. Ricci, "Group detectors for DS-CDMA systems with multipath fading channels," in Proc. Allerton Conf. Communications, Control, and Computing, Monticello, IL, Oct. 2000, pp. 816-825.

[23] J. Luo, K. Pattipati, P. Willet, and G. Levchuk, "Optimal grouping algorithm for a group detection feedback detector in synchronous CDMA communications," IEEE Trans. Commun, vol. 51, pp. 341-346, Mar. 2003.

[24] M. Varanasi and B. Aazhang, "Near-optimum demodulation for coherent communications in asynchronous gaussian CDMA channels," in Proc. CISS, Mar. 1988, pp. 832-839.

[25] — "Probability of error comparison of linear and iterative multiuser detectors," in Proc. of ComCon 88, LA, Oct. 1988.

[26] _ - "Multi-stage detection for code-division multiple access communications," IEEE Trans. Info. Th., vol. 38, Apr. 1990.

[27] R. Kohno, H. Imai, and M. Hatori, "Cancellation techniques of co-channel interference in asynchronous spread spectrum multiple access systems," Electron. Commun. in Japan.(English version), vol. 66, no. 5, pp. 20-29, May 1983.

[28] C. Hegarty and B. Vojcic, "Two-stage multiuser detection for non-coherent CDMA," in Proc.33 rd Allerton Conf. Communication, Control and Computing, Oct. 1995, pp. 1063-1072.

[29] V. Ghazi-Moghadam, L. Nelson, and M. Kaveh, "Parallel interference cancellation for CDMA systems," in Proc. 33 rd Allerton Conf. Communication, Control and Computing, Monticello, IL, Oct. 1995, pp. 216-224. 
[30] Z. Shi, W. Du, and P. Driessen, "A new multistage detector for synchronous CDMA communications," IEEE Trans. Commun., vol. 44, pp. 538-541, May 1996.

[31] R. Buehrer and B. Woerner, "Analysis of adaptive multistage interference cancellation for CDMA using an improved gaussian approximation,” IEEE Trans. Commun., vol. 44, pp. 1308-1321, Oct. 1996.

[32] X. Zhang and D. Brady, "Asymptotic multiuser efficiency for decision-directed multi-user detection," IEEE Trans. Inform. Theory, vol. 44, pp. 502-515, Mar. 1998.

[33] J. Proakis, Digital Communications, 3rd ed. McGraw Hill, New York, 1995.

[34] R. Clarke, "A statistical theory of mobile-radio reception," Bell System Tech. Jnl., vol. 47, pp. 957-1000, July-Aug 1968.

[35] W. Jakes, Microwave Mobile Communications. Wiley, New York, 1974.

[36] C. Loo, “A statistical model of land-mobile satellite link," IEEE Trans. Veh. Tech., vol. 34, no. 3, pp. 122-127, Aug 1985.

[37] B. Sklar, "Rayleigh fading channels in mobile digital communications systems-Part ii: Mitigation," IEEE Comm. Mag., vol. 35, pp. 102-112, July 1997.

[38] G. Turin, "Introduction to spread-spectrum antimultipath techniques and their application to urban digital radio," in Proc. IEEE, Mar. 1980, pp. 328-353.

[39] R. Mowbray, P. Grant, and R. Pringle, "New antimultipath technique for spread spectrum receivers," Electronics Letters, vol. 29, no. 5, p. 456:457, Aug. 1993.

[40] T. Zemen, J. Wehinger, C. F. Mecklenbrauker, and R. R. Muller, "Iterative detection and channel estimation for MCCDMA,” in Proc. IEEE International Conference on Communications (ICC), vol. 5, May 2003, pp. 3462-3466.

[41] A. Paulraj and C. Papadias, "Space-time processing for wireless communications," IEEE Sig. Proc. Mag., vol. 14, pp. 49-83, Nov.. 1997.

[42] S. Stein, "Fading channel issues in system engineering," IEEE Journal on Sel. Areas Comm., vol. 5, pp. 68-89, Feb. 1987.

[43] B. Sklar, "Rayleigh fading channels in mobile digital communications systems-Part i: Characterization," IEEE Comm. Mag., vol. 35, pp. 136-146, Sept. 1997.

[44] Y. Sanada, A. Kajiwara, and M. Nakagawa, "Adaptive rake receiver for mobile communications," IEICE Trans. Comm., vol. E76-B, p. 1002:1007, Aug.. 1993.

[45] E. Lee and D. Messerschmitt, Digital Communication. Kluwer Academic Publishers, 1994.

[46] A. Kocian and B. H. Fleury, "Em-based joint data detection and channel estimation of DS-CDMA signals," IEEE Trans. Comm., vol. 51, pp. 1709-1720, Oct. 2003.

[47] A. Sen Gupta and A. Singer, "Linear complexity multiuser detector using joint successive interference cancellation," in Asilomar Conference on Sig., Syst., and Comp., Pacific Grove, CA, Nov. 9-12 2003.

[48] J. Bucklew, "Large deviation techniques in decision, simulation and estimation”. John Wiley and Sons, 1990.

[49] J. Nelson, "Mitigating the effects of intersymbol interference: Algorithms and Analysis," Ph.D. dissertation, Univ. of Ill. at Urbana-Champaign, Oct. 2005.

[50] D. G. Luenberger, Optimization by vector space methods. John Wiley and Sons, 1988.

[51] A. Sen Gupta, "A Structural Approach to Multi-user Detection," Ph.D. dissertation, Univ. of Ill. at Urbana-Champaign, Dec. 2006. 